\title{
Trialkylstibine complexes of boron, aluminium, gallium and indium trihalides: synthesis, properties and bonding
}

\author{
Victoria K. Greenacre, William Levason and Gillian Reid ${ }^{1 *}$ \\ School of Chemistry, University of Southampton, Southampton SO17 1BJ. U.K.
}

\begin{abstract}
\end{abstract}
The reaction of $\mathrm{BX}_{3}(\mathrm{X}=\mathrm{Cl}, \mathrm{Br}$ or $\mathrm{I})$ with $\mathrm{SbR}_{3}\left(\mathrm{R}=\mathrm{Et}\right.$ or $\left.{ }^{\mathrm{i}} \mathrm{Pr}\right)$ in anhydrous hexane or toluene produced moisture sensitive $\left[\mathrm{BX}_{3}\left(\mathrm{SbR}_{3}\right)\right]$, whose stability increases with halide, $\mathrm{Cl}<\mathrm{Br}<\mathrm{I}$. Unstable $\left[\mathrm{BF}_{3}\left(\mathrm{SbR}_{3}\right)\right]$ were also characterized spectroscopically, but lose $\mathrm{BF}_{3}$ very readily. The $\left[\mathrm{MX}_{3}\left(\mathrm{SbR}_{3}\right)\right](\mathrm{M}=\mathrm{Al}, \mathrm{Ga}, \mathrm{In})$ complexes were isolated from hexane solution; the $\mathrm{Ga}$ and In complexes can be recrystallized unchanged from $\mathrm{CH}_{2} \mathrm{Cl}_{2}$, but the $\left[\mathrm{AlX}_{3}{ }_{3}\left(\mathrm{SbR}_{3}\right)\right]\left(\mathrm{X}^{\prime}=\mathrm{Br}\right.$ or I) undergo $\mathrm{X}^{\prime} / \mathrm{Cl}$ exchange with the solvent. The new complexes were characterized by IR and multinuclear NMR spectroscopy $\left({ }^{1} \mathrm{H},{ }^{13} \mathrm{C}\left\{{ }^{1} \mathrm{H}\right\},{ }^{11} \mathrm{~B},{ }^{27} \mathrm{Al},{ }^{71} \mathrm{Ga},{ }^{115} \mathrm{In}\right.$, as appropriate). $\mathrm{X}$ ray crystal structures are reported for $\left[\mathrm{BX}_{3}\left(\mathrm{SbR}_{3}\right)\right]\left(\mathrm{X}=\mathrm{Br}, \mathrm{l} ; \mathrm{R}=\mathrm{Et},{ }^{\mathrm{i}} \mathrm{Pr} ; \mathrm{X}=\mathrm{Cl}, \mathrm{R}={ }^{\mathrm{i} P r}\right),\left[\mathrm{All}_{3}\left(\mathrm{Sb}^{\mathrm{i}} \mathrm{Pr}_{3}\right)\right]$, $\left[\mathrm{GaX}_{3}\left(\mathrm{SbR}_{3}\right)\right]\left(\mathrm{X}=\mathrm{Cl}, \mathrm{I} ; \mathrm{R}={ }^{\mathrm{P}} \mathrm{Pr}\right),\left[\mathrm{GaBr}_{3}\left(\mathrm{SbEt}_{3}\right)\right]$ and $\left[\ln \mathrm{X}_{3}\left(\mathrm{SbR}_{3}\right)\right]\left(\mathrm{X}=\mathrm{Cl}, \mathrm{I} ; \mathrm{R}=\mathrm{Et}\right.$, $\left.{ }^{\mathrm{P}} \mathrm{Pr}\right)$; all contain pseudotetrahedral geometries at both the group 13 and antimony atoms, and comparisons with phosphine and arsine analogs are discussed. The donor-acceptor bond lengths are unexceptional, but coordination of the stibine is accompanied by a significant widening of the $\mathrm{C}-\mathrm{Sb}-\mathrm{C}$ angles and a small reduction in $\mathrm{C}-\mathrm{Sb}$ distances. DFT calculations confirm these result from significant changes in the antimony $5 s$ and $5 p$ contributions to the $\mathrm{Sb}-\mathrm{C}$ bonds, with corresponding increases in the $5 \mathrm{p}$ character of the antimony-based "Ione pair" on coordination. Intermolecular hypervalent $\mathrm{Cl} \cdots \mathrm{Sb}$ interactions are present in the two $\left[\operatorname{lnCl}_{3}\left(\mathrm{SbR}_{3}\right)\right]$ complexes, but absent in the others.

\section{Introduction}

For most of the twentieth century organostibine ligands were regarded as having a similar, but much more restricted coordination chemistry than their $\mathrm{P}$ or As analogs, and their study received only limited effort. ${ }^{1-4}$ Most reported complexes were of low-valent d-block metal centers, typically metal carbonyls, organometallics, or halides of the platinum metals, copper, silver, etc. Recent work has explored the complexes of the p-block elements with phosphines and arsines, but with a few exceptions, stibine complexes in this area of the Periodic Table remain unknown. ${ }^{1-5}$ However, other recent work has shown that stibines have a significantly different coordination chemistry in some systems, which have few or no analogs with the lighter pnictines. This includes the occurrence of "hypervalent" interactions, where stibines already coordinated as $\sigma$-donors to d-block metal centers simultaneously behave as $\sigma$-acceptors to other donors, including neutral amine, ether, carbonyl groups, as well as halide, triflate or fluoroborate anions. ${ }^{6-10}$ Gabbaï and co-workers have described a unique series of complexes where polydentate stibine-

\footnotetext{
${ }^{1}$ Corresponding author email G.Reid@soton.ac.uk
} 
phosphine ligands, including $\left(0-\mathrm{Ph}_{2} \mathrm{PC}_{6} \mathrm{H}_{4}\right)_{3} \mathrm{Sb}$ or $\mathrm{Ph}\left(\mathrm{o}-\mathrm{Ph}_{2} \mathrm{PC}_{6} \mathrm{H}_{4}\right)_{2} \mathrm{Sb}$, with the antimony coordinated to a platinum metal, nickel or gold center, undergo oxidation at antimony with coordination of a halogen ( $F$ or sometimes $\mathrm{Cl}$ ) to the antimony. ${ }^{5,11-14}$ Simple tertiary stibines have also been shown to behave as bridging ligands toward some d-block centers, as in $\left[\mathrm{Rh}_{2}\left(\mathrm{CPh}_{2}\right)_{2}\left(\mu_{2}-\mathrm{SbR}_{3}\right)\right]^{15}$ and $\left[\mathrm{Pd}_{4}\left(\mu_{3}-\mathrm{SbMe}_{3}\right)_{4}\left(\mathrm{~K}^{1}-\mathrm{SbMe}_{3}\right)_{4}\right]_{;}{ }^{16}$ it is notable that although $\left[\mathrm{Rh}_{2}\left(\mathrm{CPh}_{2}\right)_{2}\left(\mu_{2}-\mathrm{ER} \mathrm{R}_{3}\right)\right](\mathrm{E}=\mathrm{P} \text { ort } \mathrm{As})^{15}$ were obtained subsequently, they can only be made by metathesis of the stibine from the rhodium complex, not directly. In view of these significant differences compared to phosphine or arsine analogs, detailed exploration of stibine complexes in other areas is merited to increase our insights into the similarities and differences from the lighter analogs. Here we report the first systematic study of trialkylstibine complexes of the Group 13 metal halides using both experimental and DFT computational approaches.

The trihalides of the Group 13 elements, $\mathrm{MX}_{3}(\mathrm{M}=\mathrm{B}, \mathrm{Al}, \mathrm{Ga}, \mathrm{In} ; \mathrm{X}=\mathrm{F}, \mathrm{Cl}, \mathrm{Br}, \mathrm{I})$, exhibit varying degrees of Lewis acidity, and their coordination chemistry with neutral soft donor ligands $\left(\mathrm{PR}_{3}, \mathrm{AsR}_{3}, \mathrm{SR}_{2}, \mathrm{SeR}_{2}\right.$, etc.) has attracted considerable recent effort. ${ }^{17-19}$ These complexes are typically moisture sensitive and, for boron and aluminium, very readily hydrolysed, although much less so for the heavier metals. They are labile in solution and partially or completely decomposed by strong donor solvents. In contrast, thallium(III) halides $\operatorname{TIX}_{3}(\mathrm{X}=\mathrm{Cl}$ or $\mathrm{Br})$ are easily reduced, with halogenation of the donor atom, hence no examples with neutral soft donor ligands have been isolated. ${ }^{5,19,20}$ The Lewis acidity of the Group 13 halides varies with both $\mathrm{M}$ and $\mathrm{X}$. For boron, the Lewis acidity usually increases with $\mathrm{X}$ in the order $\mathrm{F}<\mathrm{Cl}<\mathrm{Br}<\mathrm{I}^{5,21-25}$ recent computational effort has shown this to be due to the energies of the boron $\sigma$-bonding interactions, and hence the long-held view that $B-X \pi$-back-bonding is responsible, has been discounted. ${ }^{5,21-24}$ For the other systems $(M=A l, G a, I n)$ the opposite order with $X(C l>B r>I)$ is generally found, ${ }^{5,26}$ although it should be noted that packing effects, intermolecular interactions, hydrogen bonding or interactions with solvate molecules can mask the expected trends in bond lengths within individual complexes. ${ }^{27} \mathrm{The}_{\mathrm{MF}}(\mathrm{M}=\mathrm{Al}$, $\mathrm{Ga}, \mathrm{In}$ ) are inert polymers, and although some $\mathrm{N}$ - and O-donor ligand complexes have been prepared using the $\left[\mathrm{MF}_{3}\left(\mathrm{OH}_{2}\right)_{3}\right]$ as synthons, ${ }^{28,29}$ the latter do not react with soft donor ligands, and attempted $\mathrm{Cl} / \mathrm{F}$ ligand exchange reactions on $\left[\mathrm{MCl}_{3} \mathrm{~L}_{3}\right]$ typically result in loss of the soft donor ligand $(\mathrm{L})$ and the trifluoride polymers are reformed. ${ }^{29}$

We recently reported the first X-ray structural data and detailed multinuclear spectroscopic data on selenoand telluro-ether complexes of $\mathrm{BX}_{3}{ }^{30,31}$ and $\mathrm{AlX}_{3} \cdot{ }^{32}$ Very little work has been reported on Group 13 halidestibine complexes, ${ }^{1,3,5}$ although stibine complexes of aluminium and gallium trialkyls are well established. ${ }^{4,5}$ Early studies on the $\mathrm{BX}_{3} / \mathrm{SbMe}_{3}$ system $(\mathrm{X}=\mathrm{Cl}, \mathrm{Br}$ or I) established the 1:1 stoichiometry for the adducts and presented some limited spectroscopy. ${ }^{33-35}$ It was reported that $\mathrm{BF}_{3}$ did not appear to form an adduct with $\mathrm{SbMe}_{3}{ }^{35}$ Subsequently, the synthesis and X-ray crystal structures of the thermally and photochemically unstable $\left[\mathrm{BX}_{3}\left\{\mathrm{Sb}\left(\mathrm{SiMe}_{3}\right)_{3}\right\}\right]$ were reported; ${ }^{36}$ all three complexes have $\mathrm{B}-\mathrm{Sb}$ bonds of the same length within experimental error. Two early papers mention $\mathrm{AlCl}_{3} / \mathrm{SbR}_{3}$ systems and suggest chlorination of the stibine 
occurs, but with very little supporting data presented, ${ }^{37,38}$ and the only report describing gallium or indium halide-stibine complexes appears to be that of $\left[\mathrm{GaCl}_{3}\left(\mathrm{SbMe}_{3}\right)\right]{ }^{39}$

\section{Experimental}

Infrared spectra were recorded as Nujol mulls between Csl plates using a Perkin-Elmer Spectrum 100 spectrometer over the range $4000-200 \mathrm{~cm}^{-1} \cdot{ }^{1} \mathrm{H},{ }^{13} \mathrm{C}\left\{{ }^{1} \mathrm{H}\right\},{ }^{11} \mathrm{~B},{ }^{19} \mathrm{~F}\left\{{ }^{1} \mathrm{H}\right\},{ }^{27} \mathrm{Al},{ }^{71} \mathrm{Ga}$ and ${ }^{115} \mathrm{In}$ NMR spectra were recorded from $\mathrm{CH}_{2} \mathrm{Cl}_{2} / \mathrm{CD}_{2} \mathrm{Cl}_{2}, \mathrm{CDCl}_{3}$ or $\mathrm{C}_{6} \mathrm{D}_{6}$ solutions using a Bruker $\mathrm{AV} 400$ spectrometer and referenced to TMS via the residual solvent resonance, external $\left[\mathrm{BF}_{3}\left(\mathrm{OEt}_{2}\right)\right]$, external $\mathrm{CFCl}_{3},\left[\mathrm{Al}\left(\mathrm{H}_{2} \mathrm{O}\right)_{6}\right]^{3+},\left[\mathrm{Ga}\left(\mathrm{H}_{2} \mathrm{O}\right)_{6}\right]^{3+}$ and $\left[\ln \left(\mathrm{H}_{2} \mathrm{O}\right)_{6}\right]^{3+}$ in $\mathrm{H}_{2} \mathrm{O} / \mathrm{D}_{2} \mathrm{O}$ at $\mathrm{pH}=1$, respectively. Microanalyses were undertaken at London Metropolitan University or Medac. Hexane and toluene were dried prior to use, by distillation from sodium and $\mathrm{CH}_{2} \mathrm{Cl}_{2}$ from $\mathrm{CaH}_{2}$, and all preparations were carried out under rigorously anhydrous conditions via a dry dinitrogen atmosphere and standard Schlenk and glovebox techniques. Boron trifluoride was obtained from Fluorochem, $\mathrm{BCl}_{3}, \mathrm{BBr}_{3}, \mathrm{Bl}_{3}, \mathrm{AlCl}_{3}, \mathrm{AlBr}_{3}, \mathrm{All}_{3}, \mathrm{SbPh}_{3}, \mathrm{GaCl}_{3}, \mathrm{InCl}_{3}$ and $\operatorname{InBr} r_{3}$ from Sigma-Aldrich, while $\mathrm{GaBr}_{3}$ was obtained from Alfa Aesar, $\mathrm{Gal}_{3}$ from Strem and $\mathrm{Inl}_{3}$ from Fisher; all were used as received. $\mathrm{SbEt}_{3}$ and $\mathrm{Sb}^{\mathrm{P}} \mathrm{Pr}_{3}$ were made as described. ${ }^{40} \mathrm{CAUTION}$ - some trialkylstibines are pyrophoric, care should be taken to manipulate under in strictly anaerobic conditions. Where possible, all new compounds that could be isolated were characterised by multinuclear NMR spectroscopy $\left({ }^{1} \mathrm{H},{ }^{13} \mathrm{C}\left\{{ }^{1} \mathrm{H}\right\},{ }^{11} \mathrm{~B},{ }^{19} \mathrm{~F}\left\{{ }^{1} \mathrm{H}\right\},{ }^{27} \mathrm{Al},{ }^{71} \mathrm{Ga},{ }^{115} \mathrm{In}\right.$ as appropriate), together with IR spectroscopy and elemental analysis. Single crystal X-ray studies were undertaken on representative examples and the structures determined are consistent with the other data. As described below, the compounds $\left[\mathrm{BF}_{3}\left(\mathrm{SbR}_{3}\right)\right]$ and $\left[\mathrm{BCl}_{3}\left(\mathrm{SbR}_{3}\right)\right](\mathrm{R}=\mathrm{Et}, \mathrm{i} \mathrm{Pr})$ were too unstable to isolate (fluorides) or extremely unstable (chlorides), hence spectroscopic data reported are from fresh samples prepared in situ (fluorides), or samples run immediately following isolation (chlorides). Obtaining satisfactory microanalytical data for the low-melting and extremely hydrolytically sensitive $\left[\mathrm{AlX}_{3}\left(\mathrm{Sb}^{\mathrm{i}} \mathrm{Pr}_{3}\right)\right.$ ] was not possible, however the spectroscopic data are fully consistent with their formation, and a crystal structure is reported for $\left[\mathrm{All}_{3}\left(\mathrm{Sb}^{\mathrm{i}} \mathrm{Pr}_{3}\right)\right]$.

\section{$\left[B F_{3}\left(\mathrm{SbR}_{3}\right)\right]\left(R=E t,{ }^{i} \mathrm{Pr}\right)$}

A slow stream of $\mathrm{BF}_{3}$ was bubbled through a solution of trialkylstibine (approx. $0.9 \mathrm{mmol}$ ) in $n$-hexane $(5$ $\mathrm{mL}$ ). No precipitate formed in the case of $\mathrm{SbEt}_{3}$, but a yellow oil deposited as the solvent was carefully removed in vacuo. For $\mathrm{Sb}^{i} \mathrm{Pr}_{3}$, a white precipitate formed upon addition of $\mathrm{BF}_{3}$, the solution was decanted off and the precipitate was dried briefly in vacuo, when it became pale yellow. The products fume in air and have a significant pressure of $\mathrm{BF}_{3}$ at room temperature, which prevented microanalysis from being obtained.

[BF $\left.3\left(\mathrm{SbEt}_{3}\right)\right]:{ }^{1} \mathrm{H}$ NMR (400 MHz, CD $\left.\mathrm{Cl}_{2}, 295 \mathrm{~K}\right): \delta 1.50\left(\mathrm{~s}, 6 \mathrm{H}, \mathrm{CH}_{2}\right), 1.30\left(\mathrm{~s}, 9 \mathrm{H}, \mathrm{CH}_{3}\right) .{ }^{19} \mathrm{~F}\left\{{ }^{1} \mathrm{H}\right\}(376 \mathrm{MHz}$, $\left.\mathrm{CD}_{2} \mathrm{Cl}_{2}, 295 \mathrm{~K}\right): \delta-146.4$ (s). ${ }^{11} \mathrm{~B}\left(128 \mathrm{MHz}, \mathrm{CD}_{2} \mathrm{Cl}_{2}, 295 \mathrm{~K}\right): \delta+3.0$ (s). IR (Nujol): $\mathrm{v}=1057,836 \mathrm{v}(\mathrm{B}-\mathrm{F}) ; 578$ $\delta(\mathrm{B}-\mathrm{F}) \mathrm{cm}^{-1}$. 
[BF $\left.\left.3\left(\mathbf{S b}^{\mathrm{P} P r}\right)_{3}\right)\right]:{ }^{1} \mathrm{H}$ NMR $\left(400 \mathrm{MHz}, \mathrm{CD}_{2} \mathrm{Cl}_{2}, 295 \mathrm{~K}\right): \delta 1.95(\mathrm{~s}, 3 \mathrm{H}, \mathrm{CH}), 1.35\left(\mathrm{~s}, 18 \mathrm{H}, \mathrm{CH}_{3}\right) .{ }^{19} \mathrm{~F}\left\{{ }^{1} \mathrm{H}\right\}(376 \mathrm{MHz}$, $\mathrm{CD}_{2} \mathrm{Cl}_{2}, 295 \mathrm{~K}$ ): $\delta-144.3$ (s). ${ }^{11} \mathrm{~B}\left(128 \mathrm{MHz}, \mathrm{CD}_{2} \mathrm{Cl}_{2}, 295 \mathrm{~K}\right.$ ): $\delta+4.2$ (s). IR (Nujol): $v=1057,836 \mathrm{v}(\mathrm{B}-\mathrm{F}) ; 577$ $\delta(B-F) \mathrm{cm}^{-1}$.

\section{$\left[B C l_{3}\left(\mathrm{SbR}_{3}\right)\right]\left(\mathrm{R}=\mathrm{Et},{ }^{i} \mathrm{Pr}\right)$}

A slow stream of $\mathrm{BCl}_{3}$ was bubbled through a solution of trialkylstibine $(0.202 \mathrm{~g}, 0.97 \mathrm{mmol})$ in $\mathrm{n}$-hexane $(5$ $\mathrm{mL}$ ). No precipitate formed in the case of $\mathrm{SbEt}_{3}$, but a pale brown oil deposited as the solvent was carefully removed in vacuo. For $\mathrm{Sb}^{\mathrm{P}} \mathrm{Pr}_{3}$, a white precipitate formed upon addition of $\mathrm{BCl}_{3}$, the solution was decanted off and the precipitate was dried briefly in vacuo.

[ $\left.\mathrm{BCl}_{3}\left(\mathrm{SbEt}_{3}\right)\right]$ : The NMR spectra in both $\mathrm{CD}_{2} \mathrm{Cl}_{2}$ and $\mathrm{C}_{6} \mathrm{D}_{6}$ showed rapid decomposition with significant new resonances growing in the ${ }^{1} \mathrm{H}$ and ${ }^{13} \mathrm{C}\left\{{ }^{1} \mathrm{H}\right\}$ spectra, although only a single species was present in the ${ }^{11} \mathrm{~B}$ spectrum. ${ }^{1} \mathrm{H}$ NMR $\left(400 \mathrm{MHz}, \mathrm{C}_{6} \mathrm{D}_{6}, 295 \mathrm{~K}\right.$ ): $\delta 1.22$ (quartet, $6 \mathrm{H},{ }^{3} \mathrm{~J}_{\mathrm{H}-\mathrm{H}}=7.7 \mathrm{~Hz}, \mathrm{CH}_{2}$ ), $0.98\left(\mathrm{t}, 9 \mathrm{H},{ }^{3} \mathrm{~J}_{\mathrm{H}-\mathrm{H}}=7.7 \mathrm{~Hz}\right.$, $\left.\mathrm{CH}_{3}\right) .{ }^{13} \mathrm{C}\left\{{ }^{1} \mathrm{H}\right\}$ NMR $\left(100 \mathrm{MHz}, \mathrm{C}_{6} \mathrm{D}_{6}, 295 \mathrm{~K}\right): \delta 10.15\left(\mathrm{CH}_{2}\right), 5.5\left(\mathrm{CH}_{3}\right) .{ }^{11} \mathrm{~B}$ NMR $\left(128 \mathrm{MHz}, \mathrm{C}_{6} \mathrm{D}_{6}, 295 \mathrm{~K}\right): \delta+11.0$. IR (Nujol): $v=728,665(B-C l) \mathrm{cm}^{-1}$.

[ $\left.\mathrm{BCl}_{3}\left(\mathrm{Sb}^{\mathrm{P}} \mathrm{Pr}_{3}\right)\right]$ : The flocculent white solid turned brown upon storage in a glove box, precluding microanalytical measurements. The NMR sample in $\mathrm{C}_{6} \mathrm{D}_{6}$ also began to degrade rapidly ( $<10$ minutes), revealing a mixture of $\left[\mathrm{BCl}_{3}\left(\mathrm{Sb}^{\mathrm{i}} \mathrm{Pr}_{3}\right)\right]$ and an additional boron-free species, along with a black sticky precipitate. Freshly prepared sample: ${ }^{1} \mathrm{H}$ NMR $\left(400 \mathrm{MHz}, \mathrm{C}_{6} \mathrm{D}_{6}, 295 \mathrm{~K}\right): \delta 2.02(\mathrm{br}, 3 \mathrm{H}, \mathrm{CH}), 1.20$ (br s, 18H, $\left.\mathrm{CH}_{3}\right) .{ }^{13} \mathrm{C}\left\{{ }^{1} \mathrm{H}\right\}$ NMR $\left(100 \mathrm{MHz}, \mathrm{C}_{6} \mathrm{D}_{6}, 295 \mathrm{~K}\right): \delta 21.4\left(\mathrm{CH}_{3}\right), 21.2(\mathrm{CH}) .{ }^{11} \mathrm{~B} N M R\left(128 \mathrm{MHz}, \mathrm{C}_{6} \mathrm{D}_{6}, 295 \mathrm{~K}\right): \delta$ 12.0. IR (Nujol): v $=730,620(\mathrm{~B}-\mathrm{Cl}) \mathrm{cm}^{-1}$.

\section{$\left[\mathrm{BBr}_{3}\left(\mathrm{SbR}_{3}\right)\right]$ and $\left[\mathrm{B} \mathrm{I}_{3}\left(\mathrm{SbR}_{3}\right)\right]\left(\mathrm{R}=\mathrm{Et},{ }^{i} \mathrm{Pr}\right)$}

Solutions of trialkystibines ( $\mathrm{SbEt}_{3}$ or $\mathrm{SbiPr}_{3}$ ) in $n$-hexane were typically added to a stirring solution or suspension of the corresponding $\mathrm{BX}_{3}(\mathrm{X}=\mathrm{Br}, \mathrm{I})$ in a 1:1 molar ratio. A white precipitate quickly formed, and after stirring for 1-2 hours, the solution was decanted and the white solids dried under vacuum.

[BBr$\left.r_{3}\left(\mathrm{SbEt}_{3}\right)\right]$ : From a suspension of triethylstibine $(0.21 \mathrm{~g}, 1.0 \mathrm{mmol})$ and $\mathrm{BBr}_{3}(0.1 \mathrm{~mL}, 0.1 \mathrm{mmol}), 0.197 \mathrm{~g}$ (43\%) of white solid was isolated. Anal. Required $\mathrm{C}_{6} \mathrm{H}_{15} \mathrm{BBr}_{3} \mathrm{Sb}$ (459.6): $\mathrm{C}, 15.7 ; \mathrm{H}, 3.3$. Found: $\mathrm{C}, 15.6 ; \mathrm{H}, 3.4$ \%. ${ }^{1} \mathrm{H}$ NMR (400 MHz, $\mathrm{C}_{6} \mathrm{D}_{6}, 295 \mathrm{~K}$ ): $\delta 1.34$ (quartet, $6 \mathrm{H},{ }^{3} \mathrm{~J}_{\mathrm{H}-\mathrm{H}}=7.7 \mathrm{~Hz}, \mathrm{CH}_{2}$ ), $0.96\left(\mathrm{t}, 9 \mathrm{H},{ }^{3} \mathrm{H}_{\mathrm{H}-\mathrm{H}}=7.7 \mathrm{~Hz}\right.$, $\left.\mathrm{CH}_{3}\right) .{ }^{13} \mathrm{C}\left\{{ }^{1} \mathrm{H}\right\}$ NMR $\left(100 \mathrm{MHz}, \mathrm{C}_{6} \mathrm{D}_{6}, 295 \mathrm{~K}\right): \delta 10.0\left(\mathrm{CH}_{2}\right), 7.0\left(\mathrm{CH}_{3}\right) .{ }^{11} \mathrm{~B}$ NMR $\left(128 \mathrm{MHz}, \mathrm{C}_{6} \mathrm{D}_{6}, 295 \mathrm{~K}\right): \delta-$ 18.7. IR (Nujol): $v=626,602(\mathrm{~B}-\mathrm{Br}) \mathrm{cm}^{-1}$.

[BBr$\left.{ }_{3}\left(\mathrm{Sb}^{i} \mathrm{Pr}_{3}\right)\right]$ : From a suspension of triisopropylstibine $(0.10 \mathrm{~g}, 0.39 \mathrm{mmol})$ and $\mathrm{BBr}_{3}(0.1 \mathrm{~mL}, 0.1 \mathrm{mmol})$, $0.085 \mathrm{~g}(66 \%)$ of white solid was isolated. Anal. Required $\mathrm{C}_{9} \mathrm{H}_{21} \mathrm{BBr}_{3} \mathrm{Sb}$ (501.6): C, 21.5; H, 4.2. Found: $\mathrm{C}, 21.5$ $\mathrm{H}, 4.2 \% .{ }^{1} \mathrm{H}$ NMR $\left(400 \mathrm{MHz}, \mathrm{C}_{6} \mathrm{D}_{6}, 295 \mathrm{~K}\right): \delta 2.13$ (sept, 3H, $\left.{ }^{3} \mathrm{H}_{\mathrm{H}-\mathrm{H}}=7.3 \mathrm{~Hz}, \mathrm{CH}\right), 1.20\left(\mathrm{~d}, 18 \mathrm{H},{ }^{3} \mathrm{~J}_{\mathrm{H}-\mathrm{H}}=7.3 \mathrm{~Hz}\right.$, $\left.\mathrm{CH}_{3}\right) .{ }^{13} \mathrm{C}\left\{{ }^{1} \mathrm{H}\right\}$ NMR $\left(100 \mathrm{MHz}, \mathrm{C}_{6} \mathrm{D}_{6}, 295 \mathrm{~K}\right): \delta 22.5(\mathrm{CH}), 21.2\left(\mathrm{CH}_{3}\right) .{ }^{11} \mathrm{~B} N M R\left(128 \mathrm{MHz}, \mathrm{C}_{6} \mathrm{D}_{6}, 295 \mathrm{~K}\right): \delta-18.5$. IR (Nujol): v = 627, $597(\mathrm{~B}-\mathrm{Br}) \mathrm{cm}^{-1}$. 
$\left[\mathrm{Bl}_{3}\left(\mathrm{SbEt}_{3}\right)\right]$ : From a suspension of triethylstibine $(0.099 \mathrm{~g}, 0.47 \mathrm{mmol})$ and $\mathrm{Bl}_{3}(0.185 \mathrm{~g}, 0.47 \mathrm{mmol}), 0.108 \mathrm{~g}$ (84\%) of white solid was isolated. Anal. Required $\mathrm{C}_{6} \mathrm{H}_{15} \mathrm{Bl}{ }_{3} \mathrm{Sb}$ (600.6): $\mathrm{C}, 12.0 ; \mathrm{H}, 2.5$. Found: $\mathrm{C}, 12.1 ; \mathrm{H}, 2.6$ \%. ${ }^{1} \mathrm{H}$ NMR (400 MHz, $\mathrm{C}_{6} \mathrm{D}_{6}, 295 \mathrm{~K}$ ): $\delta 1.40$ (quartet, $6 \mathrm{H},{ }^{3} \mathrm{~J}_{\mathrm{H}-\mathrm{H}}=7.7 \mathrm{~Hz}, \mathrm{CH}_{2}$ ), $0.97\left(\mathrm{t}, 9 \mathrm{H},{ }^{3} \mathrm{~J}_{\mathrm{H}-\mathrm{H}}=7.7 \mathrm{~Hz}\right.$, $\left.\mathrm{CH}_{3}\right) .{ }^{13} \mathrm{C}\left\{{ }^{1} \mathrm{H}\right\} \mathrm{NMR}\left(100 \mathrm{MHz}, \mathrm{C}_{6} \mathrm{D}_{6}, 295 \mathrm{~K}\right): \delta 10.0\left(\mathrm{CH}_{2}\right), 5.89\left(\mathrm{CH}_{3}\right) .{ }^{11} \mathrm{~B}$ NMR $\left(128 \mathrm{MHz}, \mathrm{C}_{6} \mathrm{D}_{6}, 295 \mathrm{~K}\right): \delta-88.9$. IR (Nujol): $v=530,499(B-I) \mathrm{cm}^{-1}$.

$\left[\mathrm{Bl}_{3}\left(\mathrm{Sb}^{\mathrm{P} P{ }_{3}}\right)\right]$ : From suspension of triisopropylstibine $(0.095 \mathrm{~g}, 0.37 \mathrm{mmol})$ and $\mathrm{Bl}_{3}(0.148 \mathrm{~g}, 0.37 \mathrm{mmol})$, $0.103 \mathrm{~g}(57.5 \%)$ of white solid was isolated. Anal. Required $\mathrm{C}_{9} \mathrm{H}_{21} \mathrm{Bl}_{3} \mathrm{Sb}$ (642.6): C, 16.8; $\mathrm{H}, 3.3$. Found: $\mathrm{C}$, 16.9; H, 3.2 \%. ${ }^{1} \mathrm{H}$ NMR (400 MHz, $\left.\mathrm{C}_{6} \mathrm{D}_{6}, 295 \mathrm{~K}\right): \delta 2.35$ (sept, $3 \mathrm{H},{ }^{3} \mathrm{~J}_{\mathrm{H}-\mathrm{H}}=7.3 \mathrm{~Hz}, \mathrm{CH}$ ), 1.25 (d, $18 \mathrm{H},{ }^{3} \mathrm{~J}_{\mathrm{H}-\mathrm{H}}=7.3$ $\left.\mathrm{Hz}, \mathrm{CH}_{3}\right) .{ }^{13} \mathrm{C}\left\{{ }^{1} \mathrm{H}\right\}$ NMR $\left(100 \mathrm{MHz}, \mathrm{C}_{6} \mathrm{D}_{6}, 295 \mathrm{~K}\right): \delta 24.4(\mathrm{CH}), 21.4\left(\mathrm{CH}_{3}\right) .{ }^{11} \mathrm{~B}$ NMR $\left(128 \mathrm{MHz}, \mathrm{C}_{6} \mathrm{D}_{6}, 295 \mathrm{~K}\right): \delta-$ 90.7. IR (Nujol): $v=526,496(B-I) \mathrm{cm}^{-1}$.

\section{General synthesis of $\left[A I X_{3}\left(S_{B} R_{3}\right)\right]\left(R=E t,{ }^{i} \mathrm{Pr}\right)$}

Solutions of the relevant trialkystibine $\left(\mathrm{SbEt}_{3}\right.$ or $\left.\mathrm{Sb}^{\mathrm{P}} \mathrm{Pr}_{3}\right)$ in $n$-hexane were added to a stirring suspension of the corresponding $\mathrm{AlX}_{3}(\mathrm{X}=\mathrm{Cl}, \mathrm{Br}, \mathrm{l})$ in $n$-hexane in a 1:1 molar ratio. After stirring for approx. 15 minutes, solvents were removed in vacuo, allowing isolation of products as pale solids, which melted near ambient temperature. The waxy nature and the extreme sensitivity to moisture made it impossible to transfer the products quantitatively, but yields were estimated to be 40-60\%. The extreme moisture sensitivity of $\left[\mathrm{AlX}_{3}\left(\mathrm{Sb}^{\mathrm{P}} \mathrm{Pr}_{3}\right)\right]$ also led to difficulties in obtaining precise microanalysis in some cases.

[AICl $3\left(\mathrm{SbEt}_{3}\right)$ ]: From a suspension of triethylstibine $(0.230 \mathrm{~g}, 1.1 \mathrm{mmol})$ and $\mathrm{AlCl}_{3}(0.147 \mathrm{~g}, 1.1 \mathrm{mmol})$, a white solid was isolated. Anal. Required $\mathrm{C}_{6} \mathrm{H}_{15} \mathrm{AlCl}_{3} \mathrm{Sb}$ (342.3): $\mathrm{C}, 21.1 ; \mathrm{H}, 4.4$. Found: $\mathrm{C}, 21.0 ; \mathrm{H}, 4.5 \%$ \%. ${ }^{1} \mathrm{H}$ NMR (400 MHz, $\left.\mathrm{C}_{6} \mathrm{D}_{6}, 295 \mathrm{~K}\right): \delta 1.12\left(\mathrm{br}, 6 \mathrm{H}, \mathrm{CH}_{2}\right), 0.89\left(\mathrm{br}, 9 \mathrm{H}, \mathrm{CH}_{3}\right) .{ }^{13} \mathrm{C}\left\{{ }^{1} \mathrm{H}\right\} \mathrm{NMR}\left(100 \mathrm{MHz}, \mathrm{C}_{6} \mathrm{D}_{6}, 295 \mathrm{~K}\right): \delta$ $10.8\left(\mathrm{CH}_{2}\right), 4.9\left(\mathrm{CH}_{3}\right) .{ }^{27} \mathrm{Al} \mathrm{NMR}\left(104 \mathrm{MHz}, \mathrm{C}_{6} \mathrm{D}_{6}, 295 \mathrm{~K}\right): \delta$ 109.9. IR (Nujol): $v=534,490(\mathrm{Al}-\mathrm{Cl}) \mathrm{cm}^{-1}$.

[AICl $3\left(\mathrm{Sb}^{\mathrm{P} P \mathrm{Pr}_{3}}\right)$ ]: From a suspension of triisopropylstibine $(0.330 \mathrm{~g}, 1.3 \mathrm{mmol})$ and $\mathrm{AlCl}_{3}(0.175 \mathrm{~g}, 1.3 \mathrm{mmol})$, an off white sticky solid was isolated. Anal. Required $\mathrm{C}_{9} \mathrm{H}_{21} \mathrm{AlCl}_{3} \mathrm{Sb}$ (384.3): C, 28.1; $\mathrm{H}, 5.5$. Found: $\mathrm{C}, 27.1 ; \mathrm{H}$, $5.1 \% .{ }^{1} \mathrm{H}$ NMR (400 MHz, $\mathrm{C}_{6} \mathrm{D}_{6}, 295 \mathrm{~K}$ ): $\delta 1.90$ (sept, 3H, $\left.{ }^{3} \mathrm{~J}_{\mathrm{H}-\mathrm{H}}=7.3 \mathrm{~Hz}, \mathrm{CH}\right), 1.11$ (d, $18 \mathrm{H},{ }^{3} \mathrm{~J}_{\mathrm{H}-\mathrm{H}}=7.3 \mathrm{~Hz}$, $\left.\mathrm{CH}_{3}\right) .{ }^{13} \mathrm{C}\left\{{ }^{1} \mathrm{H}\right\}$ NMR (100 MHz, $\left.\mathrm{C}_{6} \mathrm{D}_{6}, 295 \mathrm{~K}\right): \delta 21.9\left(\mathrm{CH}_{3}\right), 20.6(\mathrm{CH}) .{ }^{27} \mathrm{Al}$ NMR (104 MHz, $\left.\mathrm{C}_{6} \mathrm{D}_{6}, 295 \mathrm{~K}\right): \delta 111.6$. $\mathrm{IR}\left(\right.$ Nujol): $\mathrm{v}=536(\mathrm{br})(\mathrm{Al}-\mathrm{Cl}) \mathrm{cm}^{-1}$.

[AlBr $3\left(\mathrm{SbEt}_{3}\right)$ ]: From a suspension of triethylstibine $(0.081 \mathrm{~g}, 0.38 \mathrm{mmol})$ and $\mathrm{AlBr}_{3}(0.103 \mathrm{~g}, 0.38 \mathrm{mmol})$, an off-white solid was isolated. Anal. Required $\mathrm{C}_{6} \mathrm{H}_{15} \mathrm{AlBr}_{3} \mathrm{Sb}$ (642.6): C, 15.1; H, 3.2. Found: $\mathrm{C}, 15.3 ; \mathrm{H}, 3.3 \%$ \%. ${ }^{1} \mathrm{H}$ NMR (400 MHz, $\left.\mathrm{C}_{6} \mathrm{D}_{6}, 295 \mathrm{~K}\right)$ : $\delta 1.16$ (br, 6H, CH $), 0.92$ (br s, 9H, CH $) .{ }^{13} \mathrm{C}\left\{{ }^{1} \mathrm{H}\right\}$ NMR (100 MHz, $\left.\mathrm{C}_{6} \mathrm{D}_{6}, 295 \mathrm{~K}\right)$ : $\delta 10.9\left(\mathrm{CH}_{2}\right), 5.4\left(\mathrm{CH}_{3}\right) .{ }^{27} \mathrm{Al} \mathrm{NMR}\left(104 \mathrm{MHz}, \mathrm{C}_{6} \mathrm{D}_{6}, 295 \mathrm{~K}\right): \delta$ 93.1. IR (Nujol): v = 446, $400(\mathrm{Al}-\mathrm{Br}) \mathrm{cm}^{-1}$.

[AlBr$\left.r_{3}\left(\mathrm{Sb}^{\mathrm{P} P r}\right)_{3}\right)$ ]: From a suspension of triisopropylstibine $(0.141 \mathrm{~g}, 0.56 \mathrm{mmol})$ and $\mathrm{AlBr}_{3}(0.150 \mathrm{~g}, 0.56$ mmol), an off-white sticky solid was isolated. Anal. Required $\mathrm{C}_{9} \mathrm{H}_{21} \mathrm{AlBr}_{3} \mathrm{Sb}$ (517.6): C, 20.9; $\mathrm{H}, 4.1$. Found: $\mathrm{C}$, 20.0; H, 4.2 \%. ${ }^{1} \mathrm{H}$ NMR (400 MHz, $\mathrm{C}_{6} \mathrm{D}_{6}, 295 \mathrm{~K}$ ): $\delta 1.84$ (sept, 3H, $\left.{ }^{3} \mathrm{~J}_{\mathrm{H}-\mathrm{H}}=7.1 \mathrm{~Hz}, \mathrm{CH}\right), 1.19\left(\mathrm{~d}, 18 \mathrm{H},{ }^{3} \mathrm{~J}_{\mathrm{H}-\mathrm{H}}=7.1\right.$ 
$\left.\mathrm{Hz}, \mathrm{CH}_{3}\right) \cdot{ }^{13} \mathrm{C}\left\{{ }^{1} \mathrm{H}\right\}$ NMR (100 MHz, $\left.\mathrm{C}_{6} \mathrm{D}_{6}, 295 \mathrm{~K}\right): \delta 22.1\left(\mathrm{CH}_{3}\right), 21.4(\mathrm{CH}) .{ }^{27} \mathrm{Al}$ NMR (104 MHz, $\left.\mathrm{C}_{6} \mathrm{D}_{6}, 295 \mathrm{~K}\right): \delta$ 92.1. IR (Nujol): $v=437,402(\mathrm{Al}-\mathrm{Br}) \mathrm{cm}^{-1}$.

$\left[\mathrm{All}_{3}\left(\mathrm{SbEt}_{3}\right)\right]$ : From a suspension of triethylstibine $(0.103 \mathrm{~g}, 0.49 \mathrm{mmol})$ and $\mathrm{All}_{3}(0.202 \mathrm{~g}, 0.49 \mathrm{mmol})$, a pale sticky yellow solid was isolated. Anal. Required $\mathrm{C}_{6} \mathrm{H}_{15} \mathrm{All}{ }_{3} \mathrm{Sb}$ (616.6): $\mathrm{C}, 11.7 ; \mathrm{H}, 2.5$. Found: $\mathrm{C}, 11.5 ; \mathrm{H}, 2.4$ \%. ${ }^{1} \mathrm{H}$ NMR (400 MHz, $\left.\mathrm{C}_{6} \mathrm{D}_{6}, 295 \mathrm{~K}\right): \delta 1.23\left(\mathrm{t}, 6 \mathrm{H},{ }^{3} \mathrm{~J}_{\mathrm{H}-\mathrm{H}}=7.34 \mathrm{~Hz}, \mathrm{CH}_{2}\right), 0.96\left(\mathrm{br}, 9 \mathrm{H}, \mathrm{CH}_{3}\right) .{ }^{13} \mathrm{C}\left\{{ }^{1} \mathrm{H}\right\}$ NMR $(100$ $\mathrm{MHz}, \mathrm{C}_{6} \mathrm{D}_{6}, 295 \mathrm{~K}$ ): $\delta 10.8\left(\mathrm{CH}_{2}\right), 5.6\left(\mathrm{CH}_{3}\right) .{ }^{27} \mathrm{Al} \mathrm{NMR}\left(104 \mathrm{MHz}, \mathrm{C}_{6} \mathrm{D}_{6}, 295 \mathrm{~K}\right): \delta$ 18.9. IR (Nujol): v = 336, 306 (Al-I) $\mathrm{cm}^{-1}$.

$\left[\mathrm{All}_{3}\left(\mathrm{Sb}^{\mathrm{P}} \mathrm{Pr}_{3}\right)\right]$ : From a suspension of triisopropylstibine $(0.117 \mathrm{~g}, 0.46 \mathrm{mmol})$ and $\mathrm{All}_{3}(0.190 \mathrm{~g}, 0.46 \mathrm{mmol})$, a pale sticky yellow solid was isolated. Anal. Required $\mathrm{C}_{9} \mathrm{H}_{21} \mathrm{All}{ }_{3} \mathrm{Sb}$ (600.6): $\mathrm{C}, 16.4 ; \mathrm{H}, 3.2$. Found: $\mathrm{C}, 15.9 ; \mathrm{H}$, $2.9 \% .{ }^{1} \mathrm{H}$ NMR (400 MHz, C $\left.6 \mathrm{D}_{6}, 295 \mathrm{~K}\right): \delta 1.91$ (sept, 3H, $\left.{ }^{3} \mathrm{~J}_{\mathrm{H}-\mathrm{H}}=7.1 \mathrm{~Hz}, \mathrm{CH}\right), 1.16\left(\mathrm{~d}, 18 \mathrm{H},{ }^{3} \mathrm{~J}_{\mathrm{H}-\mathrm{H}}=7.1 \mathrm{~Hz}\right.$, $\left.\mathrm{CH}_{3}\right) .{ }^{13} \mathrm{C}\left\{{ }^{1} \mathrm{H}\right\}$ NMR (100 MHz, $\left.\mathrm{C}_{6} \mathrm{D}_{6}, 295 \mathrm{~K}\right): \delta 22.1\left(\mathrm{CH}_{3}\right), 20.5(\mathrm{CH}) .{ }^{27} \mathrm{Al}$ NMR (104 MHz, $\left.\mathrm{C}_{6} \mathrm{D}_{6}, 295 \mathrm{~K}\right): \delta$ 15.4. IR (Nujol): $v=335,304$ (Al-I) $\mathrm{cm}^{-1}$.

\section{General Synthesis of $\left[\mathrm{MX}_{3}\left(\mathrm{SbEt}_{3}\right)\right]$ and $\left[\mathrm{MX} X_{3}\left(\operatorname{Sb}^{i} \mathrm{Pr}_{3}\right)\right](M=G a, I n ; X=C l, B r, I)$}

Solutions of the relevant trialkystibine ( $\mathrm{SbEt}_{3}$ or $\left.\mathrm{Sb}^{\mathrm{P}} \mathrm{Pr}_{3}\right)$ in $n$-hexane were mixed with fine suspensions of the corresponding $\mathrm{MX}_{3}(\mathrm{M}=\mathrm{Ga}, \mathrm{In}, \mathrm{X}=\mathrm{Cl}, \mathrm{Br}, \mathrm{I})$ also in $n$-hexane in a 1:1 molar ratio. A white precipitate quickly formed, and after stirring for 1-3 hours, the solution was decanted and the white solids dried under vacuum. In some cases, an additional step of extracting in $\mathrm{CH}_{2} \mathrm{Cl}_{2}$ before precipitation via the addition of $n$ hexanes was required. Typical yields were between 40 - $60 \%$

[ $\left.\mathrm{GaCl}_{3}\left(\mathrm{SbEt}_{3}\right)\right]$ : From a suspension of triethylstibine $(0.118 \mathrm{~g}, 0.56 \mathrm{mmol})$ and $\mathrm{GaCl}_{3}(0.100 \mathrm{~g}, 0.56 \mathrm{mmol})$, $0.130 \mathrm{~g}$ of white solid was isolated. Anal. Required $\mathrm{C}_{6} \mathrm{H}_{15} \mathrm{Cl}_{3} \mathrm{GaSb}$ (384.9): C, 18.7; H, 3.9. Found: $\mathrm{C}, 18.7 ; \mathrm{H}$, $4.1 \% .{ }^{1} \mathrm{H}$ NMR (400 MHz, CDCl $3,295 \mathrm{~K}$ ): $\delta 2.11$ (quartet, $6 \mathrm{H},{ }^{3} J_{\mathrm{H}-\mathrm{H}}=7.8 \mathrm{~Hz}, \mathrm{CH}_{2}$ ), $1.48\left(\mathrm{t}, 9 \mathrm{H},{ }^{3} \mathrm{~J}_{\mathrm{H}-\mathrm{H}}=7.8 \mathrm{~Hz}\right.$, $\left.\mathrm{CH}_{3}\right) .{ }^{13} \mathrm{C}\left\{{ }^{1} \mathrm{H}\right\}$ NMR $\left(100 \mathrm{MHz}, \mathrm{CDCl}_{3}, 295 \mathrm{~K}\right): \delta 10.7\left(\mathrm{CH}_{2}\right), 6.1\left(\mathrm{CH}_{3}\right) .{ }^{71} \mathrm{Ga} \mathrm{NMR}\left(122 \mathrm{MHz}, \mathrm{CH}_{2} \mathrm{Cl}_{2}, 295 \mathrm{~K}\right): \delta$ 255. IR (Nujol): $v=373,353(\mathrm{Ga}-\mathrm{Cl}) \mathrm{cm}^{-1}$.

[ $\mathrm{GaCl}_{3}\left(\mathrm{Sb}^{\mathrm{i} P r}{ }_{3}\right)$ ]: From a suspension of triisopropylstibine $(0.221 \mathrm{~g}, 0.88 \mathrm{mmol})$ and $\mathrm{GaCl}_{3}(0.155 \mathrm{~g}, 0.88$ mmol), $0.278 \mathrm{~g}$ of white solid was isolated. Anal. Required $\mathrm{C}_{9} \mathrm{H}_{21} \mathrm{Cl}_{3} \mathrm{GaSb}$ (427.0): C, 25.3; H, 5.0. Found: $\mathrm{C}$, 25.2; H, 5.0 \%. ${ }^{1} \mathrm{H}$ NMR (400 MHz, CDCl, $295 \mathrm{~K}$ ): $\delta 2.72$ (sept, 3H, ${ }^{3} \mathrm{~J}_{\mathrm{H}-\mathrm{H}}=7.3 \mathrm{~Hz}, \mathrm{CH}$ ), 1.55 (d, $18 \mathrm{H},{ }^{3} \mathrm{~J}_{\mathrm{H}-\mathrm{H}}=7.3$ $\left.\mathrm{Hz}, \mathrm{CH}_{3}\right) .{ }^{13} \mathrm{C}\left\{{ }^{1} \mathrm{H}\right\} \mathrm{NMR}\left(100 \mathrm{MHz}, \mathrm{CDCl}_{3}, 295 \mathrm{~K}\right): \delta 22.3(\mathrm{CH}), 21.8\left(\mathrm{CH}_{3}\right) .{ }^{71} \mathrm{Ga} \mathrm{NMR}\left(122 \mathrm{MHz}, \mathrm{CH}_{2} \mathrm{Cl}_{2}, 295 \mathrm{~K}\right): \delta$ 252.7. IR (Nujol): $v=379,350(\mathrm{Ga}-\mathrm{Cl}) \mathrm{cm}^{-1}$.

[ $\left.\mathrm{GaBr}_{3}\left(\mathrm{SbEt}_{3}\right)\right]$ : From a suspension of triethylstibine $(0.135 \mathrm{~g}, 0.6 \mathrm{mmol})$ and $\mathrm{GaBr}_{3}(0.200 \mathrm{~g}, 0.6 \mathrm{mmol})$, $0.130 \mathrm{~g}$ of white solid was isolated. Anal. Required $\mathrm{C}_{6} \mathrm{H}_{15} \mathrm{Br}_{3} \mathrm{GaSb}$ (518.3): C, 13.9; $\mathrm{H}, 2.9$. Found: $\mathrm{C}, 13.8 ; \mathrm{H}$, $3.0 \% .{ }^{1} \mathrm{H} \mathrm{NMR}\left(400 \mathrm{MHz}, \mathrm{CDCl}_{3}, 295 \mathrm{~K}\right): \delta 2.05$ (quartet, $6 \mathrm{H},{ }^{3} \mathrm{~J}_{\mathrm{H}-\mathrm{H}}=8.1 \mathrm{~Hz}, \mathrm{CH}_{2}$ ), $1.43\left(\mathrm{t}, 9 \mathrm{H},{ }^{3} \mathrm{H}_{\mathrm{H}-\mathrm{H}}=8.1 \mathrm{~Hz}\right.$, $\left.\mathrm{CH}_{3}\right) .{ }^{13} \mathrm{C}\left\{{ }^{1} \mathrm{H}\right\}$ NMR $\left(100 \mathrm{MHz}, \mathrm{CDCl}_{3}, 295 \mathrm{~K}\right): \delta 10.5\left(\mathrm{CH}_{2}\right), 6.24\left(\mathrm{CH}_{3}\right) .{ }^{71} \mathrm{Ga} \mathrm{NMR}\left(122 \mathrm{MHz}, \mathrm{CH}_{2} \mathrm{Cl}_{2}, 295 \mathrm{~K}\right): \delta$ 114.5. IR (Nujol): $v=279,238(\mathrm{~B}-\mathrm{Br}) \mathrm{cm}^{-1}$. 
[ $\mathrm{GaBr}_{3}\left(\mathrm{Sb}^{\mathrm{i} P \mathrm{Pr}_{3}}\right)$ ]: From a suspension of triisopropylstibine $(0.085 \mathrm{~g}, 0.34 \mathrm{mmol})$ and $\mathrm{GaBr}_{3}(0.105 \mathrm{~g}, 0.34$ mmol), $0.060 \mathrm{~g}$ of white solid was isolated. Anal. Required $\mathrm{C}_{9} \mathrm{H}_{21} \mathrm{Br}_{3} \mathrm{GaSb}$ (560.4): C, 19.3; H, 3.8. Found: $\mathrm{C}$, $18.6 \mathrm{H}, 4.0 \%$ \% ${ }^{1} \mathrm{H}$ NMR $\left(400 \mathrm{MHz}, \mathrm{CDCl}_{3}, 295 \mathrm{~K}\right.$ ): $\delta 2.85$ (sept, $\left.3 \mathrm{H},{ }^{3} \mathrm{~J}_{\mathrm{H}-\mathrm{H}}=7.3 \mathrm{~Hz}, \mathrm{CH}\right), 1.65$ (d, $18 \mathrm{H},{ }^{3} \mathrm{~J}_{\mathrm{H}-\mathrm{H}}=7.3$ $\left.\mathrm{Hz}, \mathrm{CH}_{3}\right) .{ }^{13} \mathrm{C}\left\{{ }^{1} \mathrm{H}\right\}$ NMR $\left(100 \mathrm{MHz}, \mathrm{CDCl}_{3}, 295 \mathrm{~K}\right): \delta 22.8(\mathrm{CH}), 21.75\left(\mathrm{CH}_{3}\right) .{ }^{71} \mathrm{Ga} \mathrm{NMR}\left(122 \mathrm{MHz}, \mathrm{CH}_{2} \mathrm{Cl}_{2}, 295 \mathrm{~K}\right)$ : $\delta$ 113. IR (Nujol): $v=279,236(\mathrm{~B}-\mathrm{Br}) \mathrm{cm}^{-1}$.

[Gal ${ }_{3}\left(\mathrm{SbEt}_{3}\right)$ ]: From a suspension of triethylstibine $(0.079 \mathrm{~g}, 0.24 \mathrm{mmol})$ and $\mathrm{Gal}_{3}(0.110 \mathrm{~g}, 0.24 \mathrm{mmol})$, $0.166 \mathrm{~g}$ of white solid was isolated. Anal. Required $\mathrm{C}_{6} \mathrm{H}_{15} \mathrm{Gal}_{3} \mathrm{Sb}$ (659.3): C, 10.9; $\mathrm{H}, 2.3$. Found: $\mathrm{C}, 10.9 ; \mathrm{H}$, 2.3\%. ${ }^{1} \mathrm{H}$ NMR $\left(400 \mathrm{MHz}, \mathrm{CDCl}_{3}, 295 \mathrm{~K}\right): \delta 2.13$ (quartet, $6 \mathrm{H},{ }^{3} \mathrm{H}_{\mathrm{H}-\mathrm{H}}=8.1 \mathrm{~Hz}, \mathrm{CH}_{2}$ ), $1.54\left(\mathrm{t}, 9 \mathrm{H},{ }^{3} \mathrm{H}_{\mathrm{H}-\mathrm{H}}=8.1 \mathrm{~Hz}\right.$, $\left.\mathrm{CH}_{3}\right) .{ }^{13} \mathrm{C}\left\{{ }^{1} \mathrm{H}\right\}$ NMR $\left(100 \mathrm{MHz}, \mathrm{CDCl}_{3}, 295 \mathrm{~K}\right): \delta 10.5\left(\mathrm{CH}_{2}\right), 6.24\left(\mathrm{CH}_{3}\right) .{ }^{71} \mathrm{Ga}$ NMR $\left(122 \mathrm{MHz}, \mathrm{CH}_{2} \mathrm{Cl}_{2}, 295 \mathrm{~K}\right)$ : $\delta-247.5$. IR (Nujol): $v=235,228(\mathrm{Ga}-\mathrm{I}) \mathrm{cm}^{-1}$.

[Gal ${ }_{3}\left(\mathrm{Sb}^{\mathrm{i}} \mathrm{Pr}_{3}\right)$ ]: From a suspension of triisopropylstibine $(0.1 .9 \mathrm{~g}, 0.43 \mathrm{mmol})$ and $\mathrm{Gal}_{3}(0.196 \mathrm{~g}, 0.42 \mathrm{mmol})$, $0.220 \mathrm{~g}$ of white solid was isolated. Yield: $0.220 \mathrm{~g}$, 74 \%. Anal. Required $\mathrm{C}_{9} \mathrm{H}_{21} \mathrm{Gal}_{3} \mathrm{Sb}$ (701.4): C, 15.4; $\mathrm{H}, 3.0$. Found: C, 15.3; H, $2.9 \% .{ }^{1} \mathrm{H}$ NMR (400 MHz, CDCl, $295 \mathrm{~K}$ ): $\delta 2.93$ (sept, 3H, ${ }^{3} \mathrm{H}_{\mathrm{H}-\mathrm{H}}=7.3 \mathrm{~Hz}, \mathrm{CH}$ ), 1.69 (d, $\left.18 \mathrm{H},{ }^{3} \mathrm{~J}_{\mathrm{H}-\mathrm{H}}=7.3 \mathrm{~Hz}, \mathrm{CH}_{3}\right) .{ }^{13} \mathrm{C}\left\{{ }^{1} \mathrm{H}\right\} \operatorname{NMR}\left(100 \mathrm{MHz}, \mathrm{CDCl}_{3}, 295 \mathrm{~K}\right): \delta 23.4(\mathrm{CH}), 21.75\left(\mathrm{CH}_{3}\right) .{ }^{71} \mathrm{Ga} \operatorname{NMR}(122 \mathrm{MHz}$, $\mathrm{CH}_{2} \mathrm{Cl}_{2}, 295 \mathrm{~K}$ ): $\delta-257$. IR (Nujol): $v=230$ (br) $(\mathrm{Ga}-\mathrm{I}) \mathrm{cm}^{-1}$.

[ $\left.\operatorname{lnCl}_{3}\left(\mathrm{SbEt}_{3}\right)\right]$ : From a suspension of triethylstibine $(0.150 \mathrm{~g}, 0.72 \mathrm{mmol})$ and $\mathrm{InCl}_{3}(0.53 \mathrm{~g}, 0.24 \mathrm{mmol}), 0.040$ g of white solid was isolated. Anal. Required $\mathrm{C}_{6} \mathrm{H}_{15} \mathrm{Cl}_{3} \operatorname{lnSb}(430.0): \mathrm{C}, 16.7 ; \mathrm{H}, 3.5$. Found: $\mathrm{C}, 16.8 \mathrm{H}, 3.4 .{ }^{1} \mathrm{H}$ $\operatorname{NMR}\left(400 \mathrm{MHz}, \mathrm{CD}_{2} \mathrm{Cl}_{2}, 295 \mathrm{~K}\right): \delta 2.23$ (quartet, $\left.6 \mathrm{H},{ }^{3} \mathrm{~J}_{\mathrm{H}-\mathrm{H}}=7.8 \mathrm{~Hz}, \mathrm{CH}_{2}\right), 1.50\left(\mathrm{t}, 9 \mathrm{H},{ }^{3} \mathrm{~J}_{\mathrm{H}-\mathrm{H}}=7.8 \mathrm{~Hz}, \mathrm{CH}_{3}\right) .{ }^{13} \mathrm{C}\left\{{ }^{1} \mathrm{H}\right\}$ NMR (100 MHz, CD $\mathrm{Cl}_{2}, 295 \mathrm{~K}$ ): $\delta 10.9\left(\mathrm{CH}_{2}\right), 7.5\left(\mathrm{CH}_{3}\right) .{ }^{115} \mathrm{In} \mathrm{NMR} \mathrm{(87} \mathrm{MHz,} \mathrm{CD}_{2} \mathrm{Cl}_{2}, 295 \mathrm{~K}$ ): $\delta$ n.o. IR (Nujol): v $=318,301(\mathrm{In}-\mathrm{Cl}) \mathrm{cm}^{-1}$.

[ $\operatorname{lnCl}_{3}\left(\mathrm{Sb}^{\mathrm{PPr}}{ }_{3}\right)$ ]: From a suspension of triisopropylstibine $(0.303 \mathrm{~g}, 1.2 \mathrm{mmol})$ and $\mathrm{InCl}_{3}(0.089 \mathrm{~g}, 0.4 \mathrm{mmol})$, $0.097 \mathrm{~g}$ of white solid was isolated. Anal. Required $\mathrm{C}_{9} \mathrm{H}_{21} \mathrm{Cl}_{3} \operatorname{lnSb}(472.1): \mathrm{C}, 22.9 ; \mathrm{H}, 4.5$. Found: $\mathrm{C}, 22.8 ; \mathrm{H}$, $4.5 \% .{ }^{1} \mathrm{H}$ NMR $\left(400 \mathrm{MHz}, \mathrm{CD}_{2} \mathrm{Cl}_{2}, 295 \mathrm{~K}\right): \delta 2.94(\mathrm{br}, 3 \mathrm{H}, \mathrm{CH}), 1.63\left(\mathrm{~d}, 18 \mathrm{H},{ }^{3} \mathrm{H}_{\mathrm{H}-\mathrm{H}}=7.3 \mathrm{~Hz}, \mathrm{CH}_{3}\right) .{ }^{13} \mathrm{C}\left\{{ }^{1} \mathrm{H}\right\} \mathrm{NMR}$ (100 MHz, CD $\mathrm{Cl}_{2}, 295 \mathrm{~K}$ ): $\delta 23.9(\mathrm{CH}), 22.0\left(\mathrm{CH}_{3}\right) .{ }^{115} \mathrm{In} \mathrm{NMR}\left(87 \mathrm{MHz}, \mathrm{CH}_{2} \mathrm{Cl}_{2}, 295 \mathrm{~K}\right.$ ): n.o. IR (Nujol): $v=334$, $320(\mathrm{In}-\mathrm{Cl}) \mathrm{cm}^{-1}$.

[ $\operatorname{InBr}_{3}\left(\mathrm{SbEt}_{3}\right)$ ]: From a suspension of triethylstibine $(0.109 \mathrm{~g}, 0.52 \mathrm{mmol})$ and $\operatorname{lnBr}_{3}(0.093 \mathrm{~g}, 0.26 \mathrm{mmol})$, $0.060 \mathrm{~g}$ of white solid was isolated. Anal. Required $\mathrm{C}_{6} \mathrm{H}_{15} \mathrm{Br} 3 \mathrm{lnSb}(563.4): \mathrm{C}, 12.8 ; \mathrm{H}, 2.7$. Found: $\mathrm{C}, 12.7 ; \mathrm{H}$, $2.7 \% .{ }^{1} \mathrm{H}$ NMR (400 MHz, CD ${ }_{2} \mathrm{Cl}_{2}, 295 \mathrm{~K}$ ): $\delta 2.20$ (quartet, $6 \mathrm{H},{ }^{3} \mathrm{~J}_{\mathrm{H}-\mathrm{H}}=7.8 \mathrm{~Hz}, \mathrm{CH}_{2}$ ), $1.50\left(\mathrm{t}, 9 \mathrm{H},{ }^{3} \mathrm{H}-\mathrm{H}=7.8 \mathrm{~Hz}\right.$, $\left.\mathrm{CH}_{3}\right) .{ }^{13} \mathrm{C}\left\{{ }^{1} \mathrm{H}\right\}$ NMR $\left(100 \mathrm{MHz}, \mathrm{CD}_{2} \mathrm{Cl}_{2}, 295 \mathrm{~K}\right): \delta 11.4\left(\mathrm{CH}_{2}\right), 7.9\left(\mathrm{CH}_{3}\right) .{ }^{115}$ In NMR $\left(87 \mathrm{MHz}, \mathrm{CH}_{2} \mathrm{Cl}_{2}, 295 \mathrm{~K}\right): \delta 279$ $\left(w_{1 / 2}=2160 \mathrm{~Hz}\right) \cdot \mathrm{IR}($ Nujol)$): v=216(\mathrm{sh}), 210(\mathrm{In}-\mathrm{Br}) \mathrm{cm}^{-1}$.

[ $\operatorname{InBr}_{3}\left(\mathrm{Sb}^{\left.\mathrm{P} P \mathrm{Pr}_{3}\right)}\right)$ : From a suspension of triisopropylstibine $(0.303 \mathrm{~g}, 1.2 \mathrm{mmol})$ and $\operatorname{lnBr}_{3}(0.089 \mathrm{~g}, 0.4 \mathrm{mmol})$, $0.097 \mathrm{~g}$ of white solid was isolated. Anal. Required $\mathrm{C}_{9} \mathrm{H}_{21} \mathrm{Br}_{3} \operatorname{lnSb}$ (605.5): C 17.8; H, 3.5. Found: $\mathrm{C}, 17.8 ; \mathrm{H}$, $3.6 \% .{ }^{1} \mathrm{H}$ NMR $\left(400 \mathrm{MHz}, \mathrm{CD}_{2} \mathrm{Cl}_{2}, 295 \mathrm{~K}\right): \delta 2.94(\mathrm{br}, 3 \mathrm{H}, \mathrm{CH}), 1.63\left(\mathrm{~d}, 18 \mathrm{H},{ }^{3} \mathrm{~J}_{\mathrm{H}-\mathrm{H}}=7.3 \mathrm{~Hz}, \mathrm{CH}_{3}\right) .{ }^{13} \mathrm{C}\left\{{ }^{1} \mathrm{H}\right\} \mathrm{NMR}$ 
(100 MHz, CD $\left.\mathrm{Cl}_{2}, 295 \mathrm{~K}\right): \delta 23.9(\mathrm{CH}), 22.0\left(\mathrm{CH}_{3}\right) .{ }^{115} \mathrm{In} \mathrm{NMR}\left(87 \mathrm{MHz}, \mathrm{CH}_{2} \mathrm{Cl}_{2}, 295 \mathrm{~K}\right): \delta 285\left(\mathrm{w}_{1 / 2}=1170 \mathrm{~Hz}\right)$. IR (Nujol): v = 236, $213(\mathrm{In}-\mathrm{Br}) \mathrm{cm}^{-1}$.

[ $\left.\operatorname{lnl}_{3}\left(\mathrm{SbEt}_{3}\right)\right]$ : From a suspension of triethylstibine $(0.148 \mathrm{~g}, 0.71 \mathrm{mmol})$ and $\operatorname{lnl}_{3}(0.353 \mathrm{~g}, 0.71 \mathrm{mmol}), 0.281$ g of white solid was isolated. Anal. Required $\mathrm{C}_{6} \mathrm{H}_{15} \mathrm{I}_{3} \operatorname{lnSb}$ (704.4): $\mathrm{C}, 10.2 ; \mathrm{H}, 2.2$. Found: $\mathrm{C}, 10.3 ; \mathrm{H}, 2.2 \%{ }^{1} \mathrm{H}$ NMR (400 MHz, CDCl, $295 \mathrm{~K}$ ): $\delta 2.16$ (quartet, $6 \mathrm{H}, 3^{3} \mathrm{H}_{\mathrm{H}-\mathrm{H}}=7.8 \mathrm{~Hz}, \mathrm{CH}_{2}$ ), $1.54\left(\mathrm{t}, 9 \mathrm{H}, \mathrm{t}, 3^{3} \mathrm{H}-\mathrm{H}=7.8 \mathrm{~Hz}\right.$, $\left.\mathrm{CH}_{3}\right) .{ }^{13} \mathrm{C}\left\{{ }^{1} \mathrm{H}\right\}$ NMR $\left(100 \mathrm{MHz}, \mathrm{CDCl}_{3}, 295 \mathrm{~K}\right): \delta 10.9\left(\mathrm{CH}_{2}\right), 6.7\left(\mathrm{CH}_{3}\right) .{ }^{115} \mathrm{In} \mathrm{NMR}\left(87 \mathrm{MHz}, \mathrm{CH}_{2} \mathrm{Cl} 2,295 \mathrm{~K}\right)$ : $\delta-256\left(w_{1 / 2}=3660 \mathrm{~Hz}\right)$.

[ $\operatorname{lnl}_{3}\left(\mathbf{S b}{ }^{i} \mathbf{P r}_{3}\right)$ ]: From a suspension of triisopropylstibine $(0.150 \mathrm{~g}, 0.59 \mathrm{mmol})$ and $\operatorname{lnl}_{3}(0.296 \mathrm{~g}, 0.59 \mathrm{mmol})$, $0.131 \mathrm{~g}$ of white solid was isolated. Anal. Required $\mathrm{C}_{9} \mathrm{H}_{21} 1_{3} \operatorname{lnSb}(746.45)$ : C, 14.5; H, 2.8. Found: $\mathrm{C}, 14.3 ; \mathrm{H}$, 3.0 \%. ${ }^{1} \mathrm{H} \mathrm{NMR}\left(400 \mathrm{MHz}, \mathrm{CDCl}_{3}, 295 \mathrm{~K}\right): \delta 2.96$ (sept, $\left.3 \mathrm{H},{ }^{3} \mathrm{~J}_{\mathrm{H}-\mathrm{H}}=7.3 \mathrm{~Hz}, \mathrm{CH}\right), 1.68\left(\mathrm{~d}, 18 \mathrm{H}, \mathrm{d}, 3_{\mathrm{H}-\mathrm{H}}=7.3 \mathrm{~Hz}\right.$, $\left.\mathrm{CH}_{3}\right) .{ }^{13} \mathrm{C}\left\{{ }^{1} \mathrm{H}\right\} \mathrm{NMR}\left(100 \mathrm{MHz}, \mathrm{CDCl}_{3}, 295 \mathrm{~K}\right): \delta 23.6(\mathrm{CH}), 22.1\left(\mathrm{CH}_{3}\right) .{ }^{115} \mathrm{In} \mathrm{NMR}\left(87 \mathrm{MHz}, \mathrm{CH}_{2} \mathrm{Cl}_{2}, 295 \mathrm{~K}\right): \delta$ $-261\left(w_{1 / 2}=1770 \mathrm{~Hz}\right)$.

$S b P h_{3} / B X_{3}$ in situ reactions: Solutions of $\mathrm{SbPh}_{3}$ in $\mathrm{CD}_{2} \mathrm{Cl}_{2}$ or $\mathrm{C}_{6} \mathrm{D}_{6}$ were mixed with solutions of the corresponding $\mathrm{BX}_{3}(\mathrm{X}=\mathrm{I}$ or $\mathrm{Br})$ in a 1:1 molar ratio in $5 \mathrm{~mm}$ o.d. NMR tubes in a dry-box. For the $\mathrm{BF}_{3}$ and $\mathrm{BCl}_{3}$ reactions, the gaseous boron halide was bubbled into solutions of $\mathrm{SbPh}_{3}$ in $\mathrm{CD}_{2} \mathrm{Cl}_{2}$ or $\mathrm{C}_{6} \mathrm{D}_{6}$. Some reactions were also conducted on a preparative scale in Schlenk tubes and the products sampled by NMR spectroscopy. The NMR spectra were recorded immediately after mixing; the boron solutions typically rapidly darkened over time due to decomposition. The ${ }^{1} \mathrm{H}$ and ${ }^{13} \mathrm{C}\left\{{ }^{1} \mathrm{H}\right\}$ NMR spectra were also recorded, but were uninformative other than typically showing multiple phenyl environments.

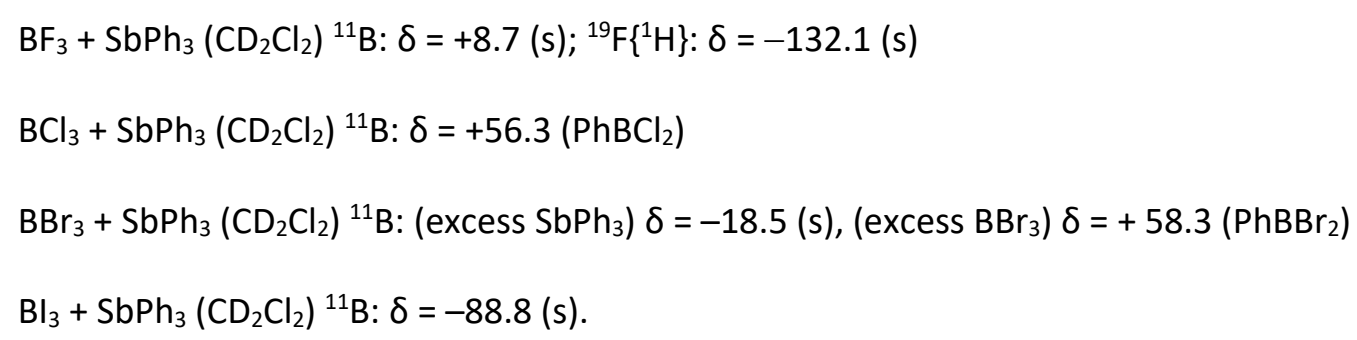

X-ray experimental: Crystals of the complexes were grown from $\mathrm{CH}_{2} \mathrm{Cl}_{2}$ solutions of the complexes allowed to evaporate slowly in the glove box. Data collections used a Rigaku AFC12 goniometer equipped with an enhanced sensitivity (HG) Saturn724+ detector mounted at the window of an FR-E+ SuperBright molybdenum $(\lambda=0.71073 \AA$ ) rotating anode generator with VHF Varimax optics (70 micron focus) with the crystal held at $100 \mathrm{~K}$, or a Agilent Xcalibur Gemini S diffractometer with a CCD plate detector using Mo-K $\alpha$ $(\lambda=0.71073 \AA)$ radiation with the crystal held at $100 \mathrm{~K}$. Structure solution and refinement were performed using SHELX(S/L)97, SHELX-2013 or SHELX-2014/7. ${ }^{41}$ Some minor disorder of 'Pr groups was evident in a few cases and therefore restraints were used. In $\left[\operatorname{lnCl}_{3}\left(\mathrm{Sb}^{\mathrm{i}} \mathrm{Pr}_{3}\right)\right]$ the disorder within one $\operatorname{Pr}$ group was modelled satisfactorily using split $\mathrm{C}$ atom site occupancies. $\mathrm{H}$ atoms bonded to $\mathrm{C}$ were placed in calculated 
positions using the default $\mathrm{C}-\mathrm{H}$ distance, and refined using a riding model. Details of the crystallographic parameters are given in the Supporting Information (Table S1). CCDC reference numbers for the crystallographic information files in cif format are: $\left.\left[\mathrm{InCl}_{3}\left(\mathrm{Sb}^{\mathrm{P} P r}\right)_{3}\right)\right]$ 1835329, $\left[\mathrm{GaBr}_{3}\left(\mathrm{SbEt}_{3}\right)\right]$ 1835330,

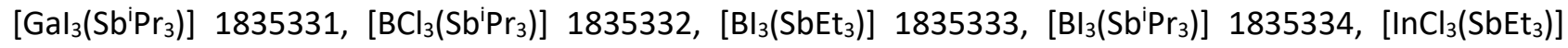

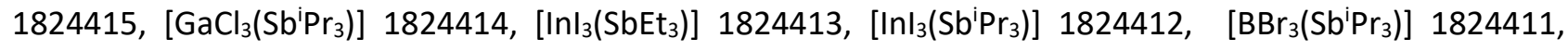
$\left[\mathrm{BBr}_{3}\left(\mathrm{SbEt}_{3}\right)\right]$ 1824410, $\left[\mathrm{All}_{3}\left(\mathrm{Sb}^{\mathrm{i}} \mathrm{Pr}_{3}\right)\right]$ 1838607. These can be obtained free of charge via www.ccdc.cam.ac.uk/data_request/cif, or by emailing data_request@ccdc.cam.ac.uk, or by contacting The Cambridge Crystallographic Data Centre, 12 Union Road, Cambridge CB2 1EZ, UK; fax: +44 1223336033.

Computational Experimental: Density functional theory (DFT) calculations (full geometry optimizations) were carried out on $\left.\left[\mathrm{BCl}_{3}\left(\mathrm{Sb}^{\mathrm{P} P r}\right)_{3}\right)\right]\left[\mathrm{BBr}_{3}\left(\mathrm{SbR}_{3}\right)\right]$, [Bl $\left.3\left(\mathrm{SbR}_{3}\right)\right]$, [All $\left.3\left(\mathrm{Sb}^{\mathrm{i}} \mathrm{Pr}_{3}\right)\right],\left[\mathrm{GaCl}_{3}\left(\mathrm{Sb}^{\mathrm{i}} \mathrm{Pr}_{3}\right)\right],\left[\mathrm{GaBr}_{3}\left(\mathrm{SbEt}_{3}\right)\right]$, $\left[\mathrm{Gal}_{3}\left(\mathrm{Sb}^{\mathrm{P}} \mathrm{Pr}_{3}\right)\right],\left[\operatorname{lnCl}_{3}\left(\mathrm{SbR}_{3}\right)\right]$ and $\left[\operatorname{lnl}_{3}\left(\mathrm{SbR}_{3}\right)\right]\left(\mathrm{R}=\mathrm{Et}\right.$ or $\left.{ }^{\mathrm{P} r}\right)$, starting from their respective crystal structure geometries with the Gaussian09 ${ }^{42}$ program and visualized using GaussView 5.0. Calculations were also run from other starting geometries and converged to the same geometries as from X-ray structures. Geometry optimization and frequency calculations were performed on the isolated molecules, and using the B3LYP43 hybrid density functional, using a basis set with a Stuttgart-Dresden effective core potential on $\mathrm{Ga}$, In, Sb, B and I atoms, with double zeta basis set on $\mathrm{H}, \mathrm{C}, \mathrm{Al}$ and $\mathrm{Cl}$ atoms. Energy minima were confirmed by the absence of any imaginary frequencies. The optimized structures were also subjected to natural bond orbital $(\mathrm{NBO})^{44}$ analysis at the same level of theory as the optimized structures.

The stibines, $\mathrm{SbEt}_{3}$ and $\mathrm{Sb}^{\mathrm{P}} \mathrm{Pr}_{3}$, were optimized starting from their geometries in the $\left[\mathrm{BBr}_{3}\left(\mathrm{SbR}_{3}\right)\right](\mathrm{R}=\mathrm{Et}$ or iPr) complex, without the $\mathrm{BBr}_{3}$ unit.

\section{Results and Discussion}

It is appropriate to discuss the synthesis and properties of the complexes with each Group 13 acceptor first, DFT exploration of the bonding and comparisons are reserved for later.

Boron Complexes: The addition of anhydrous $n$-hexane solutions of $\mathrm{SbR}_{3}\left(\mathrm{R}=\mathrm{Et}\right.$ or $\left.{ }^{\mathrm{i}} \mathrm{Pr}\right)$ to a solution of $\mathrm{BBr}_{3}$ or a suspension of powdered $\mathrm{Bl}_{3}$ in the same solvent, led to precipitation of white $\left[\mathrm{BX}_{3}\left(\mathrm{SbR}_{3}\right)\right]$ complexes. All four complexes are very moisture sensitive and the solids darken on keeping over several days at room temperature, the iodides becoming orange and the bromides brown. However the solids had sufficient stability for microanalyses, full spectroscopic data and X-ray crystal structures to be obtained for all four complexes. The complexes with $\mathrm{BCl}_{3}$ and $\mathrm{BF}_{3}$ proved to be much less stable. Bubbling $\mathrm{BCl}_{3}$ into an $n$-hexane solution of $\mathrm{Sb}^{i} \mathrm{Pr}_{3}$ led to precipitation of $\left[\mathrm{BCl}_{3}\left(\mathrm{Sb}^{i} \mathrm{Pr}_{3}\right)\right]$ as a white powder. However using $\mathrm{SbEt}_{3}$ did not yield a precipitate, but a colorless oil, identified as $\left[\mathrm{BCl}_{3}\left(\mathrm{SbEt}_{3}\right)\right]$ was obtained on careful removal of the solvent in vacuo. Both complexes of $\mathrm{BCl}_{3}$ degraded in a few hours in the solid state and more rapidly in solution in $\mathrm{CD}_{2} \mathrm{Cl}_{2}$ or $\mathrm{C}_{6} \mathrm{D}_{6}$, turning brown and then black, which precluded obtaining microanalyses; however, spectroscopic data were obtained from freshly prepared samples. Fortuitously, one synthesis of 
$\left[\mathrm{BCl}_{3}\left(\mathrm{Sb}^{\mathrm{i}} \mathrm{Pr}_{3}\right)\right]$ deposited small crystals directly from the synthesis solution and one of these was used to determine the structure.

Passing a slow stream of $\mathrm{BF}_{3}$ into $n$-hexane solutions of $\mathrm{SbR}_{3}\left(\mathrm{R}=\mathrm{Et}\right.$ or $\left.{ }^{\mathrm{i}} \mathrm{Pr}\right)$ precipitated white solids in very poor yields, which were identified spectroscopically as $\left[\mathrm{BF}_{3}\left(\mathrm{SbR}_{3}\right)\right]$. The solid $\left[\mathrm{BF}_{3}\left(\mathrm{SbR}_{3}\right)\right]$ fume in air and have a significant vapour pressure of $\mathrm{BF}_{3}$ at ambient temperatures. Whilst $\left[\mathrm{BF}_{3}\left(\mathrm{PMe}_{3}\right)\right]$ is a stable solid in the absence of moisture, solid $\left[\mathrm{BF}_{3}\left(\mathrm{AsMe}_{3}\right)\right]$ slowly loses $\mathrm{BF}_{3}$ at room temperature, ${ }^{25}$ and there is thus a clear trend in stability $\left[\mathrm{BF}_{3}\left(\mathrm{PMe}_{3}\right)\right]>\left[\mathrm{BF}_{3}\left(\mathrm{AsMe}_{3}\right)\right] \gg\left[\mathrm{BF}_{3}\left(\mathrm{SbR}_{3}\right)\right] .\left[\mathrm{BF}_{3}\left(\mathrm{EMe}_{2}\right)\right](\mathrm{E}=\mathrm{S}$ or Se$)$ are oils which also lose $\mathrm{BF}_{3}$ at ambient temperatures. ${ }^{30,31}$ The $\mathrm{NMR}$ spectra of the $\left[\mathrm{BF}_{3}\left(\mathrm{SbR}_{3}\right)\right]$ were obtained from $\mathrm{CD}_{2} \mathrm{Cl}_{2} / \mathrm{CH}_{2} \mathrm{Cl}_{2}$ solutions, with their ${ }^{1} \mathrm{H}$ spectra showing only broad resonances with small coordination shifts and without resolved $\mathrm{H}-\mathrm{H}$ couplings, attributable to reversible dissociation of the stibine. Support for this comes from the corresponding ${ }^{1} \mathrm{H}$ spectrum of a mixture of $\left[\mathrm{BF}_{3}\left(\mathrm{SbEt}_{3}\right)\right]+\mathrm{SbEt}_{3}$ which shows only single $\delta\left(\mathrm{CH}_{3}\right)$ and $\delta\left(\mathrm{CH}_{2}\right)$ resonances at $295 \mathrm{~K}$, but at $183 \mathrm{~K}$ separate resonances ascribed to the complex and $\mathrm{SbEt}_{3}$ are present. The ${ }^{1} \mathrm{H}$ and ${ }^{13} \mathrm{C}\left\{{ }^{1} \mathrm{H}\right\}$ NMR spectra of $\left[\mathrm{BX}_{3}\left(\mathrm{SbR}_{3}\right)\right](\mathrm{X}=\mathrm{Cl}, \mathrm{Br}$ or I) exhibit small shifts compared with the parent ligands, and the ${ }^{1} \mathrm{H}$ spectra show resolved ${ }^{3} \mathrm{~J}_{\mathrm{HH}}$ couplings. However, whilst the spectra obtained from freshly prepared $\mathrm{C}_{6} \mathrm{D}_{6}$ solutions of $\left[\mathrm{BX}_{3}\left(\mathrm{SbR}_{3}\right)\right](X=\mathrm{Br}$ or I) show only a single species, those of $\left[\mathrm{BCl}_{3}\left(\mathrm{SbR}_{3}\right)\right]$ darken rapidly and develop other resonances in the ${ }^{1} \mathrm{H}$ NMR spectra due to (unidentified) decomposition products.

The ${ }^{11} \mathrm{~B}$ chemical shifts $\left({ }^{11} \mathrm{~B} \text {, nat. abundance } 80 \%, \mathrm{I}=3 / 2, \equiv=32.08 \mathrm{MHz}, \mathrm{Q}=3.55 \times 10^{-30} \mathrm{~m}^{2}, \mathrm{R}_{\mathrm{c}}=754\right)^{45}$ of the $\left[\mathrm{BX}_{3}\left(\mathrm{SbR}_{3}\right)\right]$ are mainly influenced by the halogen present and occur at similar frequencies to those of complexes with $\mathrm{PR}_{3}, \mathrm{AsR}_{3}, \mathrm{SR}_{2}$ and $\mathrm{SeR}_{2}$. ${ }^{5,25,30-31,46-49}$ More detailed comparisons are limited by the use of different solvents in the literature reports, but suggest that for constant $X$, the resonances are to high frequency with donor center in the order $\mathrm{P}>\mathrm{As}>\mathrm{Sb}$. In contrast to the ${ }^{11} \mathrm{~B}$ and ${ }^{19} \mathrm{~F} \mathrm{NMR}$ spectra of $\left[\mathrm{BF}_{3}\left(\mathrm{PMe}_{3}\right)\right]$, which exhibit ${ }^{1} \mathrm{~J}_{\mathrm{BF}}$ coupling, but like $\left[\mathrm{BF}_{3}\left(\mathrm{AsMe}_{3}\right)\right]$, those of $\left[\mathrm{BF}_{3}\left(\mathrm{SbR}_{3}\right)\right]$ are singlets, again attributable to the rapid ligand exchange of the very weakly bound neutral ligand in solution. The IR spectra all show strong features assigned to the $\mathrm{v}\left(\mathrm{BX}_{3}\right)$ units with $\mathrm{C}_{3 \mathrm{v}}$ local symmetry at boron. ${ }^{25,30-31,46,50}$ The multinuclear NMR data of the $\left[\mathrm{BX}_{3}\left(\mathrm{SbR}_{3}\right)\right]$ obtained from freshly prepared anhydrous $\mathrm{C}_{6} \mathrm{D}_{6}$ solutions at $295 \mathrm{~K}$ are summarized in Table 1.

Table 1 Multinuclear NMR data. ${ }^{a}$

\begin{tabular}{|l|l|l|l|}
\hline complex & $\delta\left({ }^{1} \mathrm{H}\right)^{\mathrm{b}}$ & $\delta\left({ }^{\mathrm{b}} \mathrm{C}\left\{{ }^{1} \mathrm{H}\right\}\right)^{\mathrm{b}}$ & $\delta\left({ }^{11} \mathrm{~B} /{ }^{27} \mathrm{Al} /{ }^{71} \mathrm{Ga} /{ }^{115} \mathrm{In}\right)^{\mathrm{c}}$ \\
\hline$\left[\mathrm{BF}_{3}\left(\mathrm{SbEt}_{3}\right)\right]^{\mathrm{d}}$ & $1.30(\mathrm{~s}), 1.50(\mathrm{~s})$ & - & $+3.0(\mathrm{~s})$ \\
\hline$\left[\mathrm{BF}_{3}\left(\mathrm{SbiPr}_{3}\right)\right]^{\mathrm{d}}$ & $1.35(\mathrm{~s}), 1.95(\mathrm{~s})$ & - & $+4.2(\mathrm{~s})$ \\
\hline$\left[\mathrm{BCl}_{3}\left(\mathrm{SbEt}_{3}\right)\right]^{\mathrm{e}}$ & $0.98\left(\mathrm{t},{ }^{3} \mathrm{~J}_{\mathrm{H}-\mathrm{H}}=7.7 \mathrm{~Hz}\right), 1.22(\mathrm{q})$ & $5.5,10.15$, & +11.0 \\
\hline$\left.\left[\mathrm{BCl}_{3}\left(\mathrm{Sb}^{\mathrm{i} P r}\right)_{3}\right)\right]^{\mathrm{e}}$ & $1.20(\mathrm{br}), 2.02(\mathrm{br})$ & $21.2,21.4$ & +12.0 \\
\hline$\left[\mathrm{BBr}_{3}\left(\mathrm{SbEt}_{3}\right)\right]^{\mathrm{e}}$ & $0.96\left(\mathrm{t},{ }^{3} \mathrm{~J}_{\mathrm{H}-\mathrm{H}}=7.7 \mathrm{~Hz}\right), 1.34(\mathrm{q})$ & $7.0,10.1$ & -18.7 \\
\hline
\end{tabular}




\begin{tabular}{|c|c|c|c|}
\hline$\left[\mathrm{BBr}_{3}\left(\mathrm{Sb}^{\mathrm{i}} \mathrm{Pr}_{3}\right)\right]^{\mathrm{e}}$ & $1.20\left(\mathrm{~d},{ }^{3} \mathrm{~J}_{\mathrm{H}-\mathrm{H}}=7.3 \mathrm{~Hz}\right), 2.13$ (sept) & $21.2,22.5$ & -18.5 \\
\hline$\left[\mathrm{Bl}_{3}\left(\mathrm{SbEt}_{3}\right)\right]^{\mathrm{e}}$ & $0.97\left(\mathrm{t},{ }^{3} \mathrm{~J}_{\mathrm{H}-\mathrm{H}}=7.7\right) 1.40(\mathrm{q})$ & $5.9,10.0$ & -88.9 \\
\hline$\left[\mathrm{Bl}_{3}\left(\mathrm{Sb}^{\mathrm{i}} \mathrm{Pr}_{3}\right)\right]^{\mathrm{e}}$ & $1.25\left(\mathrm{~d}^{3} \mathrm{~J}_{\mathrm{H}-\mathrm{H}}=7.3\right), 2.35$ (sept) & $21.4,24.4$ & -90.7 \\
\hline$\left[\mathrm{AlCl}_{3}\left(\mathrm{Sb}^{\mathrm{i}} \mathrm{Pr}_{3}\right)\right]^{\mathrm{e}}$ & $1.11\left(\mathrm{~d},{ }^{3} \mathrm{~J}_{\mathrm{H}-\mathrm{H}}=7.3 \mathrm{~Hz}\right), 1.90$ (sept) & $20.6,21.9$ & +111.6 \\
\hline$\left[\mathrm{AlCl}_{3}\left(\mathrm{SbEt}_{3}\right)\right]^{\mathrm{e}}$ & $0.89(\mathrm{br}), 1.12(\mathrm{br})$ & $5.0,10.9$ & +109.5 \\
\hline$\left[\mathrm{AlBr}_{3}\left(\mathrm{Sb}^{\mathrm{i}} \mathrm{Pr}_{3}\right)\right]^{\mathrm{e}}$ & $1.19\left(\mathrm{~d},{ }^{3} \mathrm{~J}_{\mathrm{H}-\mathrm{H}}=7.1 \mathrm{~Hz}\right), 1.84(\mathrm{sept})$ & $21.4,22.1$ & +92.1 \\
\hline$\left[\mathrm{AlBr}_{3}\left(\mathrm{SbEt}_{3}\right)\right]^{\mathrm{e}}$ & $0.92(\mathrm{br}), 1.16(\mathrm{br})$ & $5.4,10.9$ & +93.1 \\
\hline$\left[\mathrm{All}_{3}\left(\mathrm{Sb}^{\mathrm{i}} \mathrm{Pr}_{3}\right)\right]^{\mathrm{e}}$ & $1.16\left(\mathrm{~d},{ }^{3} \mathrm{~J}_{\mathrm{H}-\mathrm{H}}=7.1 \mathrm{~Hz}\right), 1.91(\mathrm{sept})$ & $20.5,22.1$ & +15.4 \\
\hline$\left[\mathrm{All}_{3}\left(\mathrm{SbEt}_{3}\right)\right]^{\mathrm{e}}$ & $0.96\left(\mathrm{t},{ }^{3} \mathrm{~J}_{\mathrm{H}-\mathrm{H}}=7.3 \mathrm{~Hz}\right), 1.23(\mathrm{br})$ & $5.6,10.8$ & +18.9 \\
\hline$\left[\mathrm{GaCl}_{3}\left(\mathrm{SbEt}_{3}\right)\right]$ & $1.48\left(\mathrm{t},{ }^{3} \mathrm{~J}_{\mathrm{H}-\mathrm{H}}=7.8 \mathrm{~Hz}\right), 2.11(\mathrm{q})$ & $6.1,10.7$ & +255 \\
\hline$\left[\mathrm{GaCl}_{3}\left(\mathrm{Sb}^{\mathrm{i}} \mathrm{Pr}_{3}\right)\right]$ & $1.55\left(\mathrm{~d},{ }^{3} \mathrm{~J}_{\mathrm{H}-\mathrm{H}}=7.3 \mathrm{~Hz}\right), 2.72(\mathrm{sept})$ & $21.8,22.3$ & +253 \\
\hline$\left[\mathrm{GaBr}_{3}\left(\mathrm{SbEt}_{3}\right)\right]$ & $1.43\left(\mathrm{t},{ }^{3} \mathrm{~J}_{\mathrm{H}-\mathrm{H}}=8.1 \mathrm{~Hz}\right), 2.05(\mathrm{q})$ & $6.2,10.5$ & +114.5 \\
\hline$\left[\mathrm{GaBr}_{3}\left(\mathrm{Sb}^{\mathrm{i}} \mathrm{Pr}_{3}\right)\right]$ & $1.65\left(\mathrm{~d},{ }^{3} \mathrm{~J}_{\mathrm{H}-\mathrm{H}}=7.3 \mathrm{~Hz}\right), 2.85$ (sept) & $21.75,22.8$ & +113 \\
\hline$\left[\mathrm{Gal}_{3}\left(\mathrm{SbEt}_{3}\right)\right]$ & $1.54\left(\mathrm{t},{ }^{3} \mathrm{~J}_{\mathrm{H}-\mathrm{H}}=8.1 \mathrm{~Hz}\right), 2.13(\mathrm{q})$ & $6.2,10.5$ & -247.5 \\
\hline$\left[\mathrm{Gal}_{3}\left(\mathrm{Sb}^{\mathrm{i}} \mathrm{Pr}_{3}\right)\right]$ & $1.69\left(\mathrm{~d},{ }^{3} \mathrm{~J}_{\mathrm{H}-\mathrm{H}}=7.3 \mathrm{~Hz}\right), 2.93$ (sept) & $21.75,23.4$ & -257 \\
\hline$\left[\operatorname{lnCl}_{3}\left(\mathrm{SbEt}_{3}\right)\right]$ & $1.50\left(\mathrm{t},{ }^{3} \mathrm{~J}_{\mathrm{H}-\mathrm{H}}=7.8 \mathrm{~Hz}\right), 2.23(\mathrm{q})$ & $7.5,10.9$ & n.o. \\
\hline$\left[\operatorname{lnCl}_{3}\left(\mathrm{Sb}^{\mathrm{i}} \mathrm{Pr}_{3}\right)\right]$ & $1.62\left(\mathrm{~d},{ }^{3} \mathrm{~J}_{\mathrm{H}-\mathrm{H}}=7.3 \mathrm{~Hz}\right), 2.92($ sept $)$ & $22.0,23.9$ & n.o. \\
\hline$\left[\operatorname{lnBr}_{3}\left(\mathrm{SbEt}_{3}\right)\right]$ & $1.50\left(\mathrm{t},{ }^{3} \mathrm{~J}_{\mathrm{H}-\mathrm{H}}=7.8 \mathrm{~Hz}\right), 2.20(\mathrm{q})$ & $7.9,11.4$ & +279 \\
\hline$\left[\operatorname{lnBr}_{3}\left(\mathrm{Sb}^{i} \mathrm{Pr}_{3}\right)\right]$ & $1.64\left(\mathrm{~d},{ }^{3} \mathrm{~J}_{\mathrm{H}-\mathrm{H}}=7.3 \mathrm{~Hz}\right), 2.94($ sept $)$ & $22.0,23.9$ & +285 \\
\hline$\left[\operatorname{lnl}_{3}\left(\mathrm{SbEt}_{3}\right)\right]$ & $1.54\left(\mathrm{t},{ }^{3} \mathrm{~J}_{\mathrm{H}-\mathrm{H}}=7.8 \mathrm{~Hz}\right), 2.16(\mathrm{q})$ & $6.7,10.9$ & -256 \\
\hline$\left[\operatorname{lnl}_{3}\left(\mathrm{Sb}^{\mathrm{i}} \mathrm{Pr}_{3}\right)\right]$ & $1.68\left(\mathrm{~d},{ }^{3} \mathrm{~J}_{\mathrm{H}-\mathrm{H}}=7.3 \mathrm{~Hz}\right), 2.96($ sept $)$ & $21.1,23.6$ & -262 \\
\hline
\end{tabular}

a. data recorded at $295 \mathrm{~K}$ from $\mathrm{CH}_{2} \mathrm{Cl}_{2} / \mathrm{CD}_{2} \mathrm{Cl}_{2}$ solutions except e; b. referenced to external TMS; c. referenced to external $\left[\mathrm{BF}_{3}\left(\mathrm{OEt}_{2}\right)\right],\left[\mathrm{Al}\left(\mathrm{H}_{2} \mathrm{O}\right)_{6}\right]^{3+}$ in water at $\mathrm{pH}=1,\left[\mathrm{Ga}\left(\mathrm{H}_{2} \mathrm{O}\right)_{6}\right]^{3+}$ in water at $\mathrm{pH}=1,\left[\ln \left(\mathrm{H}_{2} \mathrm{O}\right)_{6}\right]^{3+}$ in water at $\mathrm{pH}=1$, as appropriate, n.o. = not observed; d. $\delta^{19} \mathrm{~F}\left[\mathrm{BF}_{3}\left(\mathrm{SbEt}_{3}\right)\right]=-146.4(\mathrm{~s}) ;\left[\mathrm{BF}_{3}\left(\mathrm{Sb}^{\mathrm{i}} \mathrm{Pr}_{3}\right)\right]=-144.3(\mathrm{~s})$; e. in $\mathrm{C}_{6} \mathrm{D}_{6}$ solution.

Data for the parent halides - $\mathrm{BF}_{3}:{ }^{19} \mathrm{~F} \mathrm{NMR}\left(\mathrm{CD}_{2} \mathrm{Cl}_{2}\right)$ : 127.8; ${ }^{11} \mathrm{~B} \mathrm{NMR}\left(\mathrm{CD}_{2} \mathrm{Cl}_{2}\right)$ : $\mathrm{BF}_{3}$ 11.03; $\mathrm{BCl}_{3} 41.9, \mathrm{BBr}_{3} 39.5, \mathrm{Bl}_{3} 5.5$ from Ref. ${ }^{47}$.

Stibines $\left(\mathrm{CD}_{2} \mathrm{Cl}_{2}\right)$ : SbEt ${ }_{3}^{1} \mathrm{H}=1.24(\mathrm{t},[3 \mathrm{H}]), 1.36(\mathrm{~m},[2 \mathrm{H}]) ;{ }^{13} \mathrm{C}\left\{{ }^{1} \mathrm{H}\right\}=5.52,11.59 . \mathrm{Sb}^{\mathrm{P} P r}{ }^{1} \mathrm{H}=1.33(\mathrm{~d},[6 \mathrm{H}]), 1.86(\mathrm{sep},[\mathrm{H}])$; ${ }^{13} \mathrm{C}\left\{{ }^{1} \mathrm{H}\right\}=17.0,22.5 .\left(\mathrm{C}_{6} \mathrm{D}_{6}\right): \mathrm{SbEt}_{3}{ }^{1} \mathrm{H}=1.20(\mathrm{t},[3 \mathrm{H}]), 1.22(\mathrm{~m},[2 \mathrm{H}]) ;{ }^{13} \mathrm{C}\left\{{ }^{1} \mathrm{H}\right\}=6.3,12.2 . \operatorname{Sb}^{\mathrm{i}} \operatorname{Pr}_{3}{ }^{1} \mathrm{H}=1.27(\mathrm{~d},[6 \mathrm{H}]), 1.69$ (sep, $[\mathrm{H}]) ;{ }^{13} \mathrm{C}\left\{{ }^{1} \mathrm{H}\right\}=17.6,23.0$.

X-ray crystal structures were obtained for five boron complexes (Figure 1). The key structural parameters are listed in Table 2. 


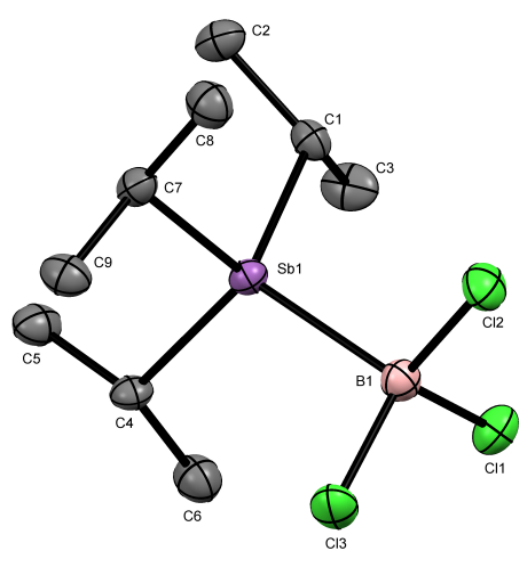

(a)

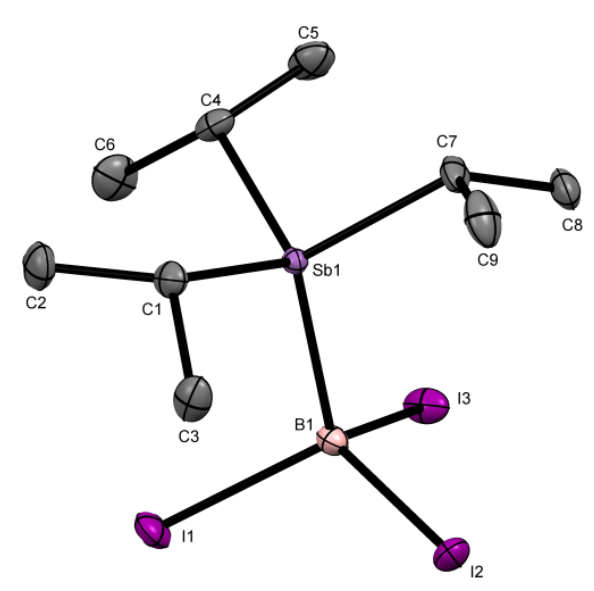

(d)

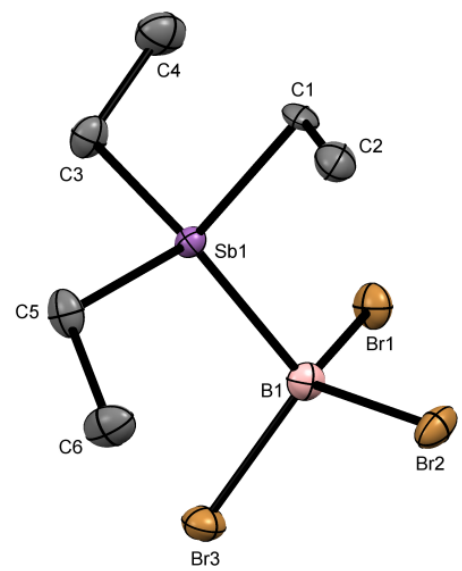

(b)

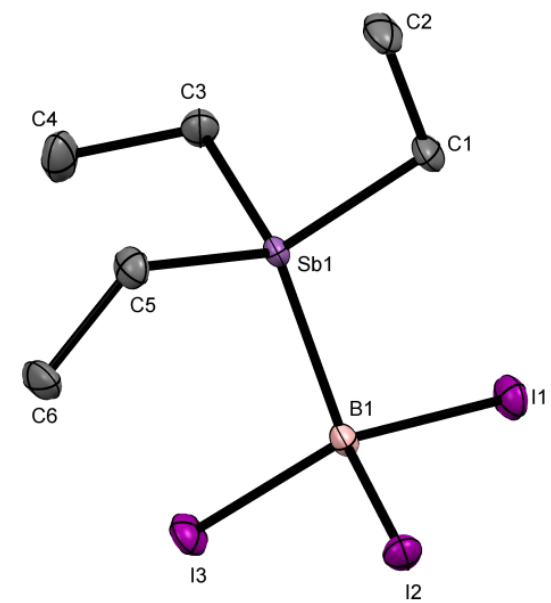

(e)

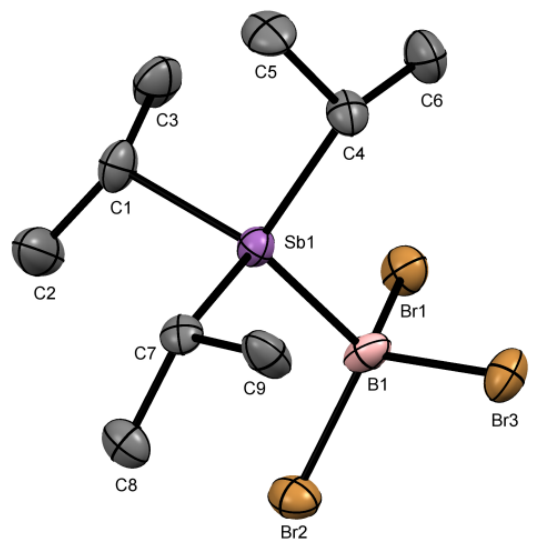

(c)

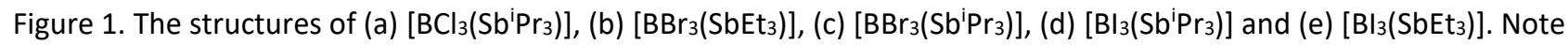
that complexes (a), (b) and (c) have a second crystallographically independent molecule in the unit cell, whilst (d) has three, all with very similar bond lengths and angles. Ellipsoids are drawn at the $50 \%$ level and hydrogen atoms omitted for clarity.

All five molecules have distorted tetrahedral arrangements about the boron and antimony and staggered geometries. The differences between the independent molecules appear to be due to solid state packing effects; there are no significant intermolecular $\mathrm{Sb} \cdots \mathrm{X}$ contacts. ${ }^{6}$

Table 2 Comparison of key bond length and angle data on the $\left[\mathrm{BX}_{3}\left(\mathrm{SbR}_{3}\right)\right]$ complexes.

\begin{tabular}{|l|l|l|l|l|}
\hline Compound & $\mathrm{d}(\mathrm{B}-\mathrm{X}) \AA$ & $\mathrm{d}(\mathrm{B}-\mathrm{Sb}) \AA$ & $\mathrm{C}-\mathrm{Sb}-\mathrm{C}^{\circ}$ & $\mathrm{d}(\mathrm{Sb}-\mathrm{C}) \AA$ \\
\hline$\left[\mathrm{BCl}\left(\mathrm{Sb}^{\mathrm{P} P r}{ }_{3}\right)\right]$ & $1.834(7)-1.843(7)$ & $2.272(7), 2.282(7)$ & $104.0(2)-110.2(2)$ & $2.140(6)-2.149(6)$ \\
\hline$\left[\mathrm{BBr}_{3}\left(\mathrm{SbEt}_{3}\right)\right]$ & $1.999(8)-2.020(8)$ & $2.266(9), 2.241(9)$ & $105.1(4)-111.8(5)$ & $2.126(8)-2.144(9)$ \\
\hline$\left[\mathrm{BBr}_{3}\left(\mathrm{Sb}^{\mathrm{i} P r_{3}}\right)\right]$ & $1.995(15)-2.026(15)$ & $2.250(13), 2.264(14)$ & $104.0(5)-110.7(5)$ & $2.142(11)-2.163(12)$ \\
\hline$\left[\mathrm{Bl}_{3}\left(\mathrm{SbEt}_{3}\right)\right]$ & $2.226(4)-2.234(4)$ & $2.230(4)$ & $104.8(2)-107.6(2)$ & $2.119(4)-2.139(4)$ \\
\hline
\end{tabular}




\begin{tabular}{|l|l|l|l|l|}
\hline$\left[\mathrm{BI}_{3}\left(\mathrm{Sb}^{\mathrm{i}} \mathrm{Pr} r_{3}\right)\right]$ & $2.222(5)-2.244(5)$ & $2.255(5)-2.256(5)$ & $104.5(2)-106.3(2)$ & $2.150(4)-2.168(4)$ \\
\hline
\end{tabular}

The $\mathrm{d}(\mathrm{B}-\mathrm{X})(\mathrm{X}=\mathrm{Cl}, \mathrm{Br}$ or $\mathrm{I})$ distances are not significantly different to those in $\left[\mathrm{BX}_{3}\left(\mathrm{PMe}_{3}\right)\right],{ }^{51}\left[\mathrm{BBr}_{3}\left(\mathrm{P}^{\mathrm{n}} \mathrm{Pr}_{3}\right)\right]{ }^{46}$ or $\left[\mathrm{BX}_{3}\left(\mathrm{AsMe}_{3}\right)\right],{ }^{52}$ whilst the increase in $\mathrm{d}(\mathrm{B}-\mathrm{E})(\mathrm{E}=\mathrm{P}, \mathrm{As}$ or $\mathrm{Sb})$ bond lengths corresponds to the increasing covalent radius of the pnictogen. ${ }^{53}$ The $d(B-S b)$ bond lengths are rather similar, but show a small decrease overall from $\mathrm{Cl}>\mathrm{Br}>\mathrm{I}$, which agrees with the predicted Lewis acidity trend of the three trihalides. ${ }^{21-24}$ The $d(B-S b)$ in the only other examples of a boron halide-stibine complexes, $\left[\mathrm{BX}_{3}\left\{\mathrm{Sb}\left(\mathrm{SiMe}_{3}\right)_{3}\right\}\right]$, are $2.259(21)$ (Cl), 2.268(17) (Br) and 2.257(8) $\AA$ (I), all are toluene solvates, ${ }^{36}$ and do not show a clear trend with X. The $\mathrm{C}-\mathrm{Sb}-\mathrm{C}$ angles in the complexes lie in the range 104-110 . Structural data for $\mathrm{SbEt}_{3}$ or $\mathrm{Sb}^{\mathrm{i}} \mathrm{Pr}_{3}$, which are liquids at room temperature, do not seem to be available, but the electron diffraction data for gaseous $\mathrm{SbMe}_{3}$ shows an average $\mathrm{C}-\mathrm{Sb}-\mathrm{C}$ angle of $94.1^{\circ}, 5^{\mathrm{S}}$ and in the $\mathrm{X}$-ray structure of $\mathrm{Sb}^{\mathrm{t}} \mathrm{Bu}_{3}$ the angles are $103.5^{\circ}$ $(\mathrm{av})^{55}$ Since the angles in $\mathrm{SbEt}_{3}$ or $\mathrm{Sb}^{\mathrm{i}} \mathrm{Pr}_{3}$ are expected to be only slightly larger than those in $\mathrm{SbMe}_{3}$, the $\mathrm{C}-\mathrm{Sb}-\mathrm{C}$ angles have opened out significantly upon coordination. Taking the $\mathrm{Sb}-\mathrm{C}$ distance in $\mathrm{SbMe}_{3}$ $(2.163(3) \AA)$ or $\mathrm{Sb}^{\mathrm{t} B u_{3}}(2.229(3) \AA)$ as a guide, and comparing these with the $\mathrm{Sb}-\mathrm{C}$ distances in the coordinated stibines suggests the latter are slightly shorter on average. This aspect is discussed in the section dealing with the DFT analysis below.

Aluminium complexes: The reaction of $\mathrm{AlX}_{3}\left(\mathrm{X}=\mathrm{Cl}, \mathrm{Br}\right.$ or I) with $\mathrm{SbEt}_{3}$ or $\mathrm{Sb}^{\mathrm{i}} \mathrm{Pr}_{3}$ in anhydrous $n$-hexane or toluene produced $\left[\mathrm{AlX}_{3}\left(\mathrm{SbR}_{3}\right)\right]$ complexes as yellow waxes or low melting solids. These are extremely moisture sensitive, the ${ }^{27} \mathrm{Al} \mathrm{NMR}\left({ }^{27} \mathrm{Al}\right.$, nat. abundance $100 \%, \mathrm{I}=5 / 2, \Xi=26.06 \mathrm{MHz}, \mathrm{Q}=0.42 \times 10^{-28} \mathrm{~m}^{2}, \mathrm{R}_{\mathrm{c}}=$ 1170) spectra showing that $\left[\mathrm{AlX}_{4}\right]^{-}$, identified by their characteristic ${ }^{27} \mathrm{Al}$ chemical shifts, ${ }^{45}$ form very readily upon trace hydrolysis. They dissolve readily in dry toluene and $C_{6} D_{6}$, and the solutions do not show any significant decomposition over some hours if protected from moisture. The chloro-complexes are soluble in $\mathrm{CH}_{2} \mathrm{Cl}_{2}$ without reaction, but $\left[\mathrm{AlX}_{3}\left(\mathrm{SbR}_{3}\right)\right](\mathrm{X}=\mathrm{Br}$ or I) react with the solvent via $\mathrm{Cl} / \mathrm{X}$ exchange, the solutions exhibiting several ${ }^{27} \mathrm{Al} N \mathrm{NMR}$ resonances due to $\left[\mathrm{AIX}_{3-\mathrm{n}} \mathrm{Cl}_{n}\left(\mathrm{SbR}_{3}\right)\right](\mathrm{n}=1-3)$; similar reactivity was encountered in the corresponding $\left[\mathrm{AIX}_{3}\left(\mathrm{ER}_{3}\right)\right](\mathrm{E}=\mathrm{P}$ or $\mathrm{As})$ systems. ${ }^{26}$ The ${ }^{27} \mathrm{Al}$ chemical shifts are dominated by the halide present (Table 1), moving to low frequency with halide $\mathrm{Cl}>\mathrm{Br}>\mathrm{I}$. Comparison with the corresponding phosphine and arsine complexes ${ }^{26,56,57}$ shows that whilst the chlorides have $\delta\left({ }^{27} \mathrm{Al}\right)$ for all three pnictine complexes within a small range ( 112-104 ppm), those of the bromides and iodides show a clear trend, with $\delta\left({ }^{27} \mathrm{Al}\right)$ moving to low frequency from $\mathrm{P}>\mathrm{As}>\mathrm{Sb}$. The $\mathrm{Al}-\mathrm{X}$ bonds weaken as Group 17 is descended, and whilst $\mathrm{Al}-\mathrm{Cl}$ bonds dominate the chemical shift in the chloro-complexes, the electronic effects of the neutral ligand become more evident in the heavier halide complexes. Contrary to suggestions in the older literature, ${ }^{37-38}$ cleavage of $\mathrm{Sb}-\mathrm{C}$ bonds was not observed in the trialkylstibine-aluminium complexes under rigorously anhydrous conditions; hydrolysis generates $\mathrm{HX}$ which is known to cleave $\mathrm{Sb}-\mathrm{C}$ bonds to form haloalkylstibines. ${ }^{1-4}$ Due to their waxy/low melting nature, X-ray quality crystals of the aluminium 
complexes proved to be difficult to obtain, but the structure of $\left[\mathrm{All}_{3}\left(\mathrm{Sb}^{\mathrm{i}} \mathrm{Pr}_{3}\right)\right]$ was determined (Table 3, Fig. 2).

Gallium and indium complexes: The reaction of $\mathrm{GaX}_{3}$ or $\ln \mathrm{X}_{3}\left(\mathrm{X}=\mathrm{Cl}, \mathrm{Br}\right.$ or I) with $\mathrm{SbR}_{3}(\mathrm{R}=\mathrm{Et}$ or $\mathrm{Pr})$ in $n$ hexane produced white 1:1 complexes, $\left[\mathrm{MX}_{3}\left(\mathrm{SbR}_{3}\right)\right]$. The solids appear reasonably air-stable, but are readily hydrolyzed in solution in organic solvents, and in contrast to the boron and aluminium complexes, can be recrystallized from $\mathrm{CH}_{2} \mathrm{Cl}_{2}$ without reaction. Although indium halides form both 4- and 5-coordinate complexes with phosphines or arsines, ${ }^{5,58-61}$ the stibines gave only 4-coordinate complexes, and addition of more $\mathrm{Sb}^{i} \mathrm{Pr}_{3}$ to solutions of $\left[\mathrm{InX}_{3}\left(\mathrm{Sb}^{\mathrm{i}} \mathrm{Pr}_{3}\right)\right]$ in $\mathrm{CH}_{2} \mathrm{Cl}_{2}$ did not change the ${ }^{115}$ In NMR spectra, showing there was no tendency to produce a higher coordination number in solution. The X-ray crystal structures of $\left[\mathrm{GaX}_{3}\left(\mathrm{Sb}^{\mathrm{i} P r_{3}}\right)\right](\mathrm{X}=\mathrm{Cl}, \mathrm{I}),\left[\mathrm{GaBr}_{3}\left(\mathrm{SbEt}_{3}\right)\right],\left[\mathrm{InCl}_{3}\left(\mathrm{SbR}_{3}\right)\right]$ and $\left[\mathrm{Inl}_{3}\left(\mathrm{SbR}_{3}\right)\right]\left(\mathrm{R}=\mathrm{Et}\right.$, $\left.{ }^{\mathrm{i}} \mathrm{Pr}\right)$ each contain distorted tetrahedral centres at antimony and the group 13 atom site (Figs. 2 and 3) with the $\left[\mathrm{GaBr}_{3}\left(\mathrm{SbEt}_{3}\right)\right]$ and $\left[\ln \mathrm{X}_{3}\left(\mathrm{SbEt}_{3}\right)\right]$ having eclipsed conformations, while the other three are staggered. The key bond length and angle data are given in Table 3.

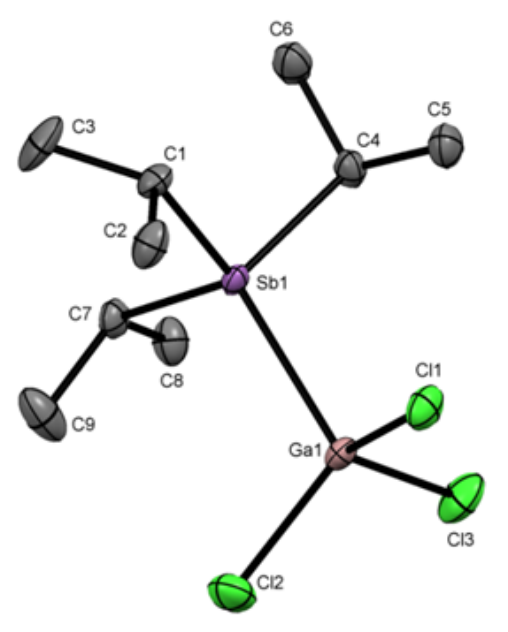

(a)

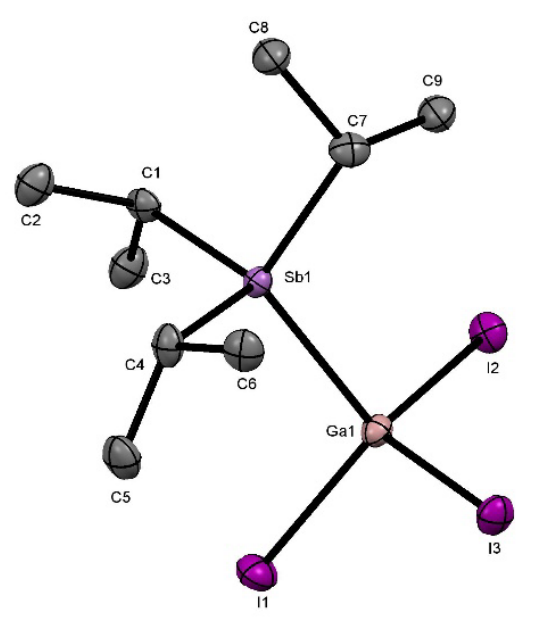

(c)

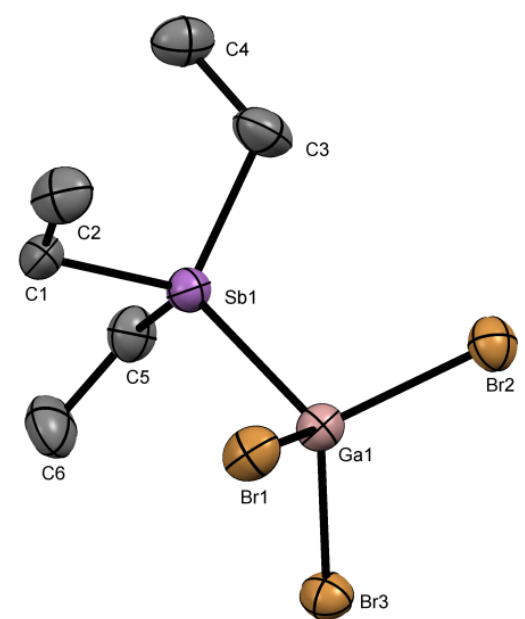

(b)

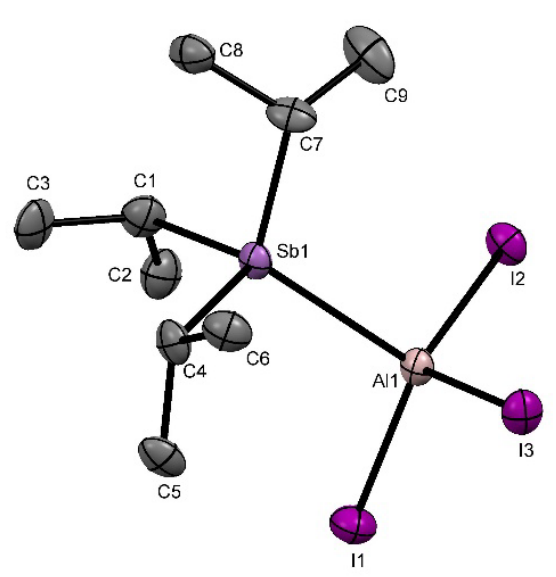

(d) 
Figure 2. The structures of (a) [ $\left.\mathrm{GaCl}_{3}\left(\mathrm{Sb}^{i} \mathrm{Pr}_{3}\right)\right]$, (b) $\left[\mathrm{GaBr}_{3}\left(\mathrm{SbEt}_{3}\right)\right]$, (c) [ $\left.\mathrm{Gal}_{3}\left(\mathrm{Sb}^{i} \mathrm{Pr}_{3}\right)\right]$, (d) [ $\left.\mathrm{All}_{3}\left(\mathrm{Sb}^{\mathrm{i}} \mathrm{Pr}_{3}\right)\right]$. Ellipsoids are drawn at the $50 \%$ level and hydrogen atoms omitted for clarity.

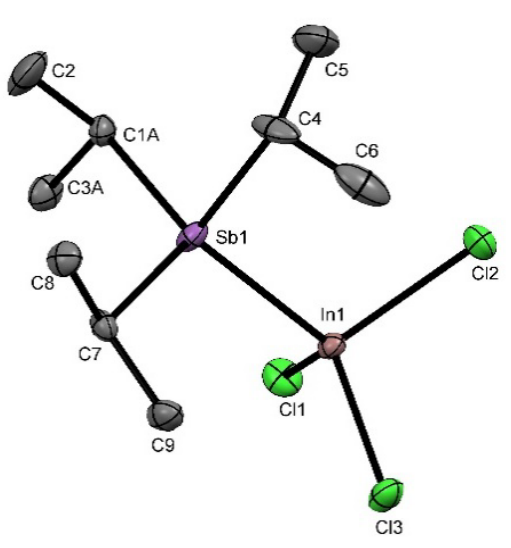

(a)

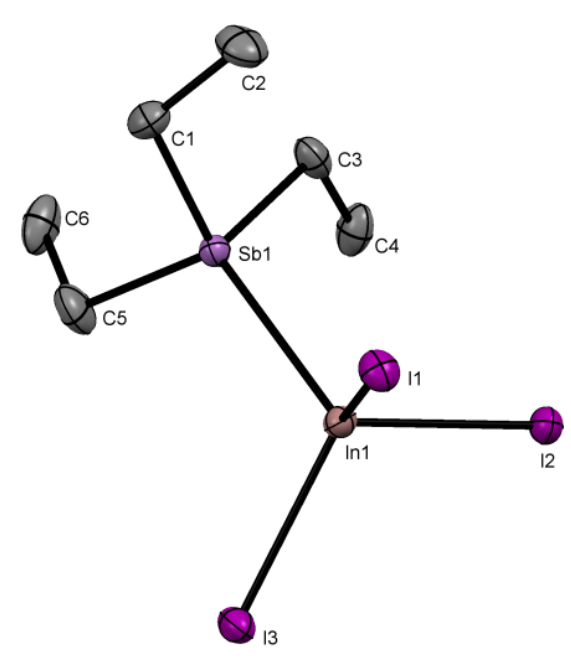

(c)

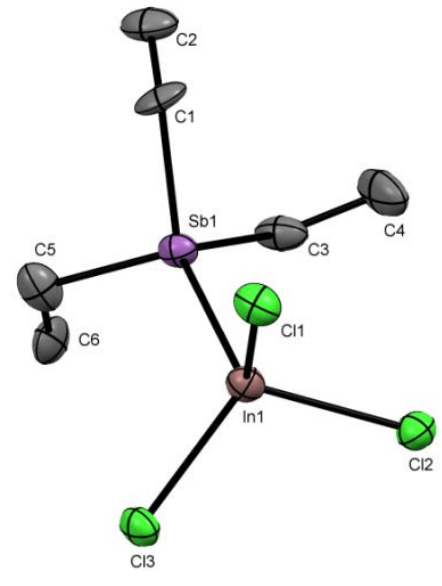

(b)

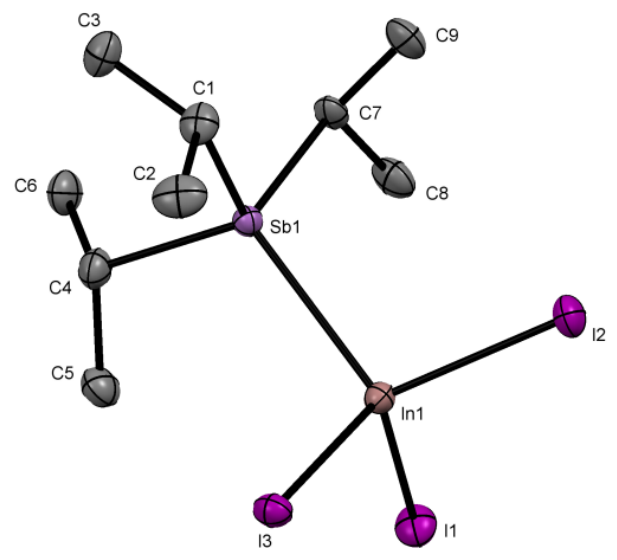

(d)

Figure 3. The structures of (a) $\left[\operatorname{InCl}_{3}\left(\mathrm{Sb}^{\mathrm{P} P P_{3}}\right)\right]$, (b) $\left[\operatorname{lnCl}_{3}\left(\mathrm{SbEt}_{3}\right)\right]$, (c) [ $\left.\operatorname{Inl}_{3}\left(\mathrm{SbEt}_{3}\right)\right]$ and (c) [ $\left.\ln _{3}\left(\mathrm{Sb}^{\mathrm{i} P \mathrm{Pr}_{3}}\right)\right]$. One $\mathrm{Pr}$ group in (a) was disordered over two sites, only one form is shown. Ellipsoids are drawn at the $50 \%$ level and hydrogen atoms omitted for clarity.

Table 3 Comparison of key bond length and angle data on the $\left[\mathrm{All}_{3}\left(\mathrm{Sb}^{i} \mathrm{Pr}_{3}\right)\right],\left[\mathrm{GaX}_{3}\left(\mathrm{SbR}_{3}\right)\right]$ and $\left[\ln X_{3}\left(\mathrm{SbR}_{3}\right)\right]$ complexes.

\begin{tabular}{|l|l|l|l|l|}
\hline Compound & $\mathrm{d}(\mathrm{Al} / \mathrm{Ga} / \mathrm{In}-\mathrm{X}) \AA$ & $\mathrm{d}(\mathrm{Al} / \mathrm{Ga} / \mathrm{In}-\mathrm{Sb}) \AA$ & $\mathrm{C}-\mathrm{Sb}-\mathrm{C}^{\circ}$ & $\mathrm{d}(\mathrm{Sb}-\mathrm{C}) \AA$ \\
\hline$\left.\left[\mathrm{All}_{3}\left(\mathrm{Sb}^{\mathrm{P} P r}\right)_{3}\right)\right]$ & $2.507(10)-2.525(9)$ & $2.680(9)$ & $102.4(8)-107.7(8)$ & $2.16(2)-2.17(2)$ \\
\hline$\left[\mathrm{GaCl}_{3}\left(\mathrm{Sb}^{\mathrm{i}} \mathrm{Pr}_{3}\right)\right]$ & $2.1730(12)-2.1811(12)$ & $2.6297(5)$ & $104.4(2)-108.0(2)$ & $2.150(4)-2.165(4)$ \\
\hline$\left[\mathrm{GaCl}_{3}\left(\mathrm{SbMe}_{3}\right)\right]^{20}$ & $2.1649(8)-2.1791(7)$ & $2.6119(4)$ & $103.7(2)-105.2(1)$ & $2.104(3)-2.111(3)$ \\
\hline$\left[\mathrm{GaBr}_{3}\left(\mathrm{SbEt}_{3}\right)\right]$ & $2.3105(13)-2.3181(12)$ & $2.6166(10)$ & $103.4(4)-106.4(4)$ & $2.108(9)-2.112(8)$ \\
\hline$\left[\mathrm{Gal}_{3}\left(\mathrm{Sb}^{\mathrm{i}} \mathrm{Pr}_{3}\right)\right]$ & $2.575(14)-2.5482(14)$ & $2.6396(14)$ & $103.4(4)-108.2(4)$ & $2.140(12)-2.151(11)$ \\
\hline$\left[\mathrm{InCl}_{3}\left(\mathrm{Sb}^{\mathrm{P} P r}\right)\right]$ & $2.3523(6)-2.3739(6)$ & $2.7772(3)$ & $105.5(1)-113.6(2)^{\mathrm{a}}$ & $2.148(7)-2.158(2)$ \\
\hline
\end{tabular}




\begin{tabular}{|l|l|l|l|l|}
\hline$\left[\operatorname{InCl}_{3}\left(\mathrm{SbEt}_{3}\right)\right]$ & $2.376(4)-2.394(4)$ & $2.7713(13)$ & $102.5(6)-104.8(6)$ & $2.111(1)-2.114(1)$ \\
\hline$\left[\operatorname{InI}_{3}\left(\mathrm{SbEt}_{3}\right)\right]$ & $2.6961(3)-2.7207(3)$ & $2.8121(3)$ & $102.2(1)-106.2(1)$ & $2.135(3)-2.137(3)$ \\
\hline$\left[\operatorname{InI}_{3}\left(\mathrm{Sb}^{\mathrm{P} P P_{3}}\right)\right]$ & $2.7000(3)-2.7124(17)$ & $2.8049(3)$ & $103.6(2)-109.2(2)$ & $2.166(4)-2.171(5)$ \\
\hline
\end{tabular}

a. Disorder of one 'Pr group was modelled using split site occupancies - see Experimental.

The $\left[\mathrm{InCl}_{3}\left(\mathrm{SbR}_{3}\right)\right]$ structures show intermolecular hypervalent $\mathrm{Sb} \cdots \mathrm{X}$ contacts ${ }^{6}$ leading to weakly associated dimers (Figure 4), but no such interactions are observed in the iodide structures.

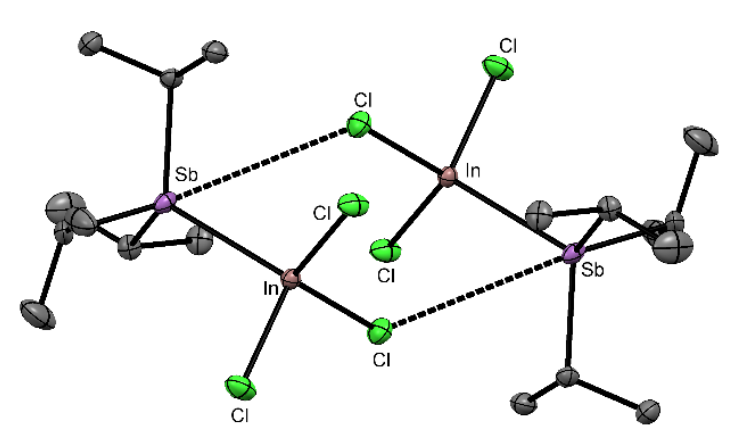

(a)

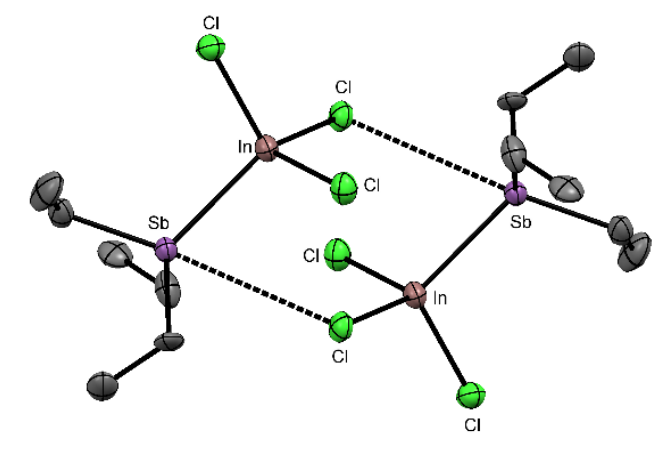

(b)

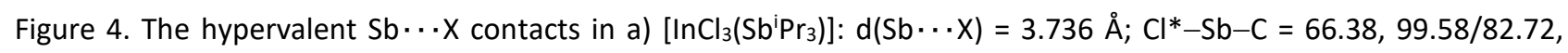
$161.7, \mathrm{Cl}^{*}-\mathrm{Sb}-\mathrm{In}=64.54^{\circ}$, and b) $\left[\operatorname{lnCl}_{3}\left(\mathrm{SbEt}_{3}\right)\right] \mathrm{d}(\mathrm{Sb} \cdots \mathrm{X})=3.654 \AA \AA^{\circ} ; \mathrm{Cl}^{*}-\mathrm{Sb}-\mathrm{C}=73.33,74.51,175.71, \mathrm{Cl}{ }^{*}-\mathrm{Sb}-\mathrm{In}=$ $71.53^{\circ}$. Ellipsoids are drawn at the $50 \%$ level and hydrogen atoms omitted for clarity. Sum of van der Waals radii $(\mathrm{Cl}+$ $\mathrm{Sb})=3.91 \AA .{ }^{.62}$ (Atoms labelled * refer to those from the adjacent molecule).

Comparison of the X-ray structures of $\left[\mathrm{GaCl}_{3}\left(\mathrm{PMe}_{3}\right)\right]^{63}\left[\mathrm{GaCl}_{3}\left(\mathrm{AsMe}_{3}\right)\right]^{64}$ and $\left[\mathrm{GaCl}_{3}\left(\mathrm{SbR}_{3}\right)\right]\left(\mathrm{R}={ }^{\mathrm{i}} \mathrm{Pr}, \mathrm{Me}^{39}\right)$

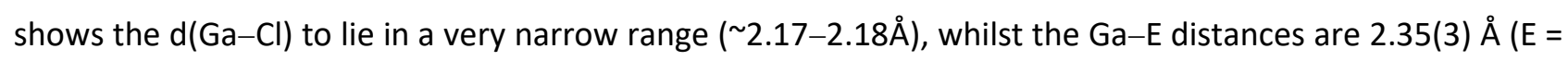
$\mathrm{P})$ and 2.433(1) $\AA$ ( $\mathrm{E}=\mathrm{As})$. The trend in $\mathrm{d}(\mathrm{Ga}-\mathrm{Sb})$ with halide is less clear; that in $\left[\mathrm{GaCl}_{3}\left(\mathrm{Sb}^{\mathrm{i}} \mathrm{Pr}_{3}\right)\right]$ appears to be out of line, possibly a packing effect. ${ }^{27}$ As noted for the boron cases above, the $\mathrm{C}-\mathrm{Sb}-\mathrm{C}$ angles increase significantly on coordination to the $\mathrm{Ga}$ or In halides and the $\mathrm{Sb}-\mathrm{C}$ distances shorten slightly, showing this is a general feature of Group 13 stibine complexes, and not due to the significantly different size and orbital energies of boron.

The structures of $\left[\operatorname{lnCl}_{3}\left(\mathrm{SbR}_{3}\right)\right]$ and $\left[\operatorname{lnl}_{3}\left(\mathrm{SbR}_{3}\right)\right]$ show $\mathrm{d}(\mathrm{In}-\mathrm{Sb}) \sim 2.77$ and $\sim 2.81 \AA$ respectively, consistent with the chloride being the stronger Lewis acid (unfortunately all crystals of $\left[\operatorname{lnBr}_{3}\left(\mathrm{SbR}_{3}\right)\right]$ examined were twinned). The $d(\operatorname{In}-1)$ distances in $\left[\operatorname{Inl}_{3}\left(\mathrm{Sb}^{\mathrm{P}} \mathrm{Pr}_{3}\right)\right]$ and $\left[\operatorname{Inl}_{3}\left(\mathrm{PiPr}_{3}\right)\right],{ }^{65}$ which are isomorphous, are the same within experimental error.

The ${ }^{1} \mathrm{H}$ and ${ }^{13} \mathrm{C}\left\{{ }^{1} \mathrm{H}\right\}$ NMR spectra obtained from $\mathrm{CD}_{2} \mathrm{Cl}_{2}$ solutions were unexceptional (Table 1). Although ${ }^{71} \mathrm{Ga}$ is a quadrupolar nucleus $\left({ }^{71} \mathrm{Ga}\right.$ nat. abundance $40 \%, \mathrm{I}=3 / 2, \equiv=30.49 \mathrm{MHz}, \mathrm{Q}=0.112 \times 10^{-28} \mathrm{~m}^{2}, \mathrm{R}_{\mathrm{c}}=$ 
319), resonances are readily observed in many pseudo-tetrahedral complexes. ${ }^{45}$ The halide present dominates the observed chemical shifts, but comparing $\left[\mathrm{GaX}_{3} \mathrm{~L}\right]\left(\mathrm{L}=\mathrm{PR}_{3}, \mathrm{AsR}_{3}, \mathrm{SR}_{2}, \mathrm{SbR}_{3}\right)$ complexes with soft donor ligands, ${ }^{20,64,66}$ shows that whilst $\delta\left({ }^{71} \mathrm{Ga}\right)$ fall in a small $(\sim 15 \mathrm{ppm})$ range for the chloride complexes, for the bromides the range is larger, and for the iodides is $\sim 100 \mathrm{ppm}$. For the heavier halide complexes, $\delta\left({ }^{71} \mathrm{Ga}\right)$ moves to low frequency with $\mathrm{L}=\mathrm{PR}_{3}>\mathrm{AsR}_{3} \sim \mathrm{SR}_{2}>\mathrm{SbR}_{3}$. In contrast, ${ }^{115} \mathrm{In}$ has a much larger quadrupole moment $\left(115 \mathrm{I}\right.$, nat. abundance $95.7 \%, \mathrm{I}=9 / 2, \equiv=21.91 \mathrm{MHz}, \mathrm{Q}=1.16 \times 10^{-28} \mathrm{~m}^{2}, \mathrm{Rc}=$ $1890)^{45}$ and resonances are only observed in relatively high symmetry cases where the indium environment has a low electric field gradient. ${ }^{67,68}$ In the present cases the indium bromide and iodide stibine complexes exhibited broad singlets (Table 1), but no resonances were found for $\left[\operatorname{lnCl}_{3}\left(\mathrm{SbR}_{3}\right)\right]$. The mismatch in electronic properties of chloride and the stibine would be expected to lead to a significant efg and this promotes sufficiently fast relaxation to result in loss of the resonance. The $v(M-X)$ vibrations in the far IR spectra are typical of complexes of both metals of type $\left[\mathrm{MX}_{3}(\mathrm{~L})\right]{ }^{61,66,68}$

Reactions of $\mathrm{BX}_{3}$ with $\mathrm{SbPh}_{3}$ : In contrast to the successful characterisation of the trialkylstibine complexes described above, the corresponding reactions involving $\mathrm{SbPh}_{3}$ were much less successful. The danger of using $\mathrm{SbPh}_{3}$ as a "typical" stibine has been stressed before, ${ }^{1,3}$ and is clearly borne out in the present work. The reaction of $\mathrm{Bl}_{3}$ and $\mathrm{SbPh}_{3}$ in dry toluene produced a yellow solution which rapidly turned deep orange. A freshly prepared solution of the constituents in $C_{6} D_{6}$ exhibited a single ${ }^{11} B$ NMR resonance at $\delta=-88.8$, consistent with the formation of $\left[\mathrm{Bl}_{3}\left(\mathrm{SbPh}_{3}\right)\right]$. Over time this resonance decreased in intensity and multiple resonances were present in the ${ }^{1} \mathrm{H}$ and ${ }^{13} \mathrm{C}\left\{{ }^{1} \mathrm{H}\right\} \mathrm{NMR}$ spectra. A mixture of $\mathrm{BBr}_{3}$ and $\mathrm{SbPh}_{3}$ in $\mathrm{a}<1: 1 \mathrm{molar}$ ratio in $\mathrm{C}_{6} \mathrm{D}_{6}$ exhibited a sharp singlet ${ }^{11} \mathrm{~B} N \mathrm{NMR}$ resonance at $\delta=-18.5$, in the range expected for $\left[\mathrm{BBr}_{3}\left(\mathrm{SbPh}_{3}\right)\right]$ but removal of the solvent in vacuo also resulted in loss of the boron bromide. In situ mixtures of $\mathrm{SbPh}_{3}$ and an excess of $\mathrm{BBr}_{3}$ in $\mathrm{CD}_{2} \mathrm{Cl}_{2}$ solution showed a sharp ${ }^{11} \mathrm{~B} \mathrm{NMR}$ resonance at $\delta=+58.3$ and a very broad feature $12 \mathrm{ppm}$. The former can be assigned to $\mathrm{PhBBr}_{2}{ }^{47,69}$ On cooling the solution to $193 \mathrm{~K}$, the broad resonance splits into two sharp features at $\delta=+40.0$ and -17.4 , assigned to $\mathrm{BBr}_{3}{ }^{47}$ and $\left[\mathrm{BBr}_{3}\left(\mathrm{SbPh}_{3}\right)\right]$, respectively. We conclude that $\mathrm{BBr}_{3}$ and $\mathrm{SbPh}_{3}$ initially form an unstable complex, $\left[\mathrm{BBr}_{3}\left(\mathrm{SbPh}_{3}\right)\right]$, which is rapidly exchanging with excess $\mathrm{BBr}_{3}$ at ambient temperatures. The $\mathrm{PhBBr}_{2}$ must be formed by the exchange:

$$
\mathrm{BBr}_{3}+\mathrm{SbPh}_{3} \rightarrow \mathrm{PhBBr}_{2}+\mathrm{Ph}_{2} \mathrm{SbBr}
$$

The reaction of $\mathrm{BCl}_{3}$ with $\mathrm{SbPh}_{3}$ in either $\mathrm{C}_{6} \mathrm{D}_{6}$ or $\mathrm{CD}_{2} \mathrm{Cl}_{2}$ produced $\mathrm{PhBCl}_{2}$, ${ }^{47,69}$ with $\delta\left({ }^{11} \mathrm{~B}\right)=+56.3\left(\mathrm{C}_{6} \mathrm{D}_{6}\right)$ or $+58.4\left(\mathrm{CD}_{2} \mathrm{Cl}_{2}\right)$, suggesting a major reaction is analogous to that in eq. 1 , and with no NMR spectroscopic evidence for any $\left[\mathrm{BCl}_{3}\left(\mathrm{SbPh}_{3}\right)\right]$ adduct formation. The multinuclear $\mathrm{NMR}$ data from a $\mathrm{CH}_{2} \mathrm{Cl}_{2}$ solution of $\mathrm{SbPh}_{3}$ saturated with $\mathrm{BF}_{3}$ gas show low frequency shifts in the ${ }^{19} \mathrm{~F}$ and ${ }^{11} \mathrm{~B}$ resonances from the values in $\mathrm{BF}_{3}$, consistent with a weak interaction in solution. Cooling the solution to $193 \mathrm{~K}$ produced only modest low temperature shifts in the resonances. Scrambling of substituents (eq. 1) would not be expected in the fluoride system due to the very strong B-F bonds, however, attempts to isolate pure boron complexes from 
these reactions failed. The reaction of various substituted triarylstibines with $\mathrm{BCl}_{3}$ or $\mathrm{BBr}_{3}$, followed by treatment with $\mathrm{MeOH}$ and 1,3-propanediol to afford arylboronates has been described, ${ }^{70}$ but the initial formation of simple adducts was not explored.

DFT Analysis: Computational studies of trialkylstibine-boron, gallium and indium halide complexes were undertaken to supplement the experimental data. The ground state geometries of $\left[\mathrm{BCl}_{3}\left(\mathrm{Sb}^{\mathrm{i}} \mathrm{Pr}_{3}\right)\right]$, $\left[\mathrm{BBr}_{3}\left(\mathrm{SbEt}_{3}\right)\right], \quad\left[\mathrm{BBr}_{3}\left(\mathrm{Sb}^{\left.\mathrm{i} P \mathrm{Pr}_{3}\right)}\right], \quad\left[\mathrm{Bl}_{3}\left(\mathrm{SbEt}_{3}\right)\right], \quad\left[\mathrm{Bl}_{3}\left(\mathrm{Sb}^{\mathrm{i}} \mathrm{Pr}_{3}\right)\right], \quad\left[\mathrm{AlCl}_{3}\left(\mathrm{Sb}^{\mathrm{i}} \mathrm{Pr}_{3}\right)\right], \quad\left[\mathrm{GaCl}_{3}\left(\mathrm{Sb}^{\mathrm{i}} \mathrm{Pr}_{3}\right)\right], \quad\left[\mathrm{GaBr}_{3}\left(\mathrm{SbEt}_{3}\right)\right]\right.$, $\left.\left[\mathrm{Gal}_{3}\left(\mathrm{Sb}^{\mathrm{P} P r}\right)_{3}\right)\right], \quad\left[\operatorname{InCl}_{3}\left(\mathrm{SbEt}_{3}\right)\right],\left[\operatorname{InCl}_{3}\left(\mathrm{Sb}^{\mathrm{i} P P_{3}}\right)\right], \quad\left[\operatorname{Inl}_{3}\left(\mathrm{SbEt}_{3}\right)\right]$ and $\left[\operatorname{Inl}_{3}\left(\mathrm{Sb}^{\mathrm{P}} \mathrm{Pr}_{3}\right)\right]$ were optimized using DFT with the B3LYP functional and the basis set described earlier for all atoms. In each case, the starting geometry of the Group 13 complex was the solid-state structures, and the bond lengths and bond angles in the calculated ground state geometries are in close agreement with experimental data (SI, Table S4).

In addition, the structures of four uncoordinated stibines ( $\left.\mathrm{SbR}_{3} \mathrm{R}=\mathrm{Me}, \mathrm{Et},{ }^{\mathrm{i} P r},{ }^{\mathrm{t}} \mathrm{Bu}\right)$ have been calculated. The optimized structures of $\mathrm{SbMe}_{3}$ and $\mathrm{Sb}^{\mathrm{t}} \mathrm{Bu}_{3}$ were calculated based on experimental data for $\mathrm{SbMe}_{3}$ and $\mathrm{Sb}^{\mathrm{t}} \mathrm{Bu}_{3}$ available from electron diffraction ${ }^{54}$ or X-ray diffraction data, ${ }^{55}$ respectively, and compare well (SI, Table S2).

The bond lengths from the calculated stibines are similar to those from available experimental data and show that the average $\mathrm{C}-\mathrm{Sb}-\mathrm{C}$ angles from $\mathrm{SbEt}_{3}$ and $\mathrm{Sb}^{\mathrm{i}} \mathrm{Pr}_{3}$ (96.0 and $98.1^{\circ}$, respectively) are slightly wider than found in $\mathrm{SbMe}_{3}\left(94.8^{\circ}\right)$ and slightly narrower compared to those found in $\mathrm{Sb}^{\mathrm{t}} \mathrm{Bu}_{3}\left(103.4^{\circ}\right)$.

The key bond lengths for the complexes fit the trends from the X-ray data, with the $d(B-S b)$ becoming shorter from $\mathrm{Cl}>\mathrm{Br}>\mathrm{I}$, as expected. The $\mathrm{d}(\mathrm{Ga}-\mathrm{Sb})$ seem to fit the reverse trend, $\mathrm{Cl}<\mathrm{Br}<\mathrm{l}$, supporting the suggestion that the $\mathrm{Sb}-\mathrm{Ga}$ bond distance observed from the X-ray crystal structure of $\left[\mathrm{GaCl}_{3}\left(\mathrm{Sb}^{\mathrm{i}} \mathrm{Pr}_{3}\right)\right]$ is anomalous due to packing effects (or possibly some crystallographic disorder).

The $\mathrm{Sb}-\mathrm{C}$ bond distances from the DFT analysis (2.16-2.19 $\AA$ ) are similar to those from experimental data (2.11-2.17 ̊̊) (Table 4), and show a slight shortening upon coordination, compared to the 'free' ligand (Sb-C distances: $2.18 \mathrm{Me}$; $2.20 \mathrm{Et} ; 2.22 \mathrm{Pr} ; 2.26^{\mathrm{t}} \mathrm{Bu}$ ). The $\mathrm{C}-\mathrm{Sb}-\mathrm{C}$ bond angles for all complexes (102-106 ${ }^{\circ}$ ) are comparable to $\mathrm{X}$-ray data $\left(104-108^{\circ}\right.$ ), and are significantly widened (as a result of coordination) in comparison to the 'free' stibines.

Table 4 The average $\mathrm{Sb}-\mathrm{C}$ bond distances $(\AA)$ and $\mathrm{C}-\mathrm{Sb}-\mathrm{C}$ bond angles $\left({ }^{\circ}\right)$ from experimental and computational data.

\begin{tabular}{|c|c|c|c|c|}
\hline \multirow{2}{*}{} & \multicolumn{2}{|c|}{ DFT } & \multicolumn{2}{c|}{ X-Ray } \\
\cline { 2 - 5 } & $\mathrm{Sb}-\mathrm{C}$ & $\mathrm{C}-\mathrm{Sb}-\mathrm{C}$ & $\mathrm{Sb}-\mathrm{C}$ & $\mathrm{C}-\mathrm{Sb}-\mathrm{C}$ \\
\hline $\mathrm{SbEt}_{3}$ & 2.199 & 96.001 & - & - \\
\hline $\mathrm{Sb}^{\mathrm{i} P r_{3}}$ & 2.221 & 98.092 & - & - \\
\hline \hline$\left[\mathrm{BCl}_{3}\left(\mathrm{Sb}^{\mathrm{i}} \mathrm{Pr}_{3}\right)\right]$ & 2.186 & 105.919 & 2.144 & 105.55 \\
\hline$\left[\mathrm{BBr}_{3}\left(\mathrm{SbEt}_{3}\right)\right]$ & 2.159 & 104.785 & 2.133 & 107.1 \\
\hline
\end{tabular}




\begin{tabular}{|c|c|c|c|c|}
\hline$\left[\mathrm{BBr}_{3}\left(\mathrm{Sb}^{\mathrm{i}} \mathrm{Pr}_{3}\right)\right]$ & 2.192 & 103.878 & 2.152 & 105.55 \\
\hline$\left[\mathrm{Bl}_{3}\left(\mathrm{SbEt}_{3}\right)\right]$ & 2.163 & 104.725 & 2.126 & 105.88 \\
\hline$\left[\mathrm{Bl}_{3}\left(\mathrm{Sb}^{\mathrm{i}} \mathrm{Pr}_{3}\right)\right]$ & 2.196 & 103.062 & 2.157 & 105.328 \\
\hline$\left[\mathrm{All}_{3}\left(\mathrm{Sb}^{\mathrm{i}} \mathrm{Pr}_{3}\right)\right]$ & 2.193 & 105.042 & 2.17 & 104.133 \\
\hline$\left[\mathrm{GaCl}_{3}\left(\mathrm{Sb}^{\mathrm{i}} \mathrm{Pr}_{3}\right)\right]$ & 2.188 & 105.732 & 2.158 & 106.137 \\
\hline$\left[\mathrm{GaBr}_{3}\left(\mathrm{SbEt}_{3}\right)\right]$ & 2.167 & 103.487 & 2.11 & 104.5 \\
\hline$\left[\mathrm{Gal}_{3}\left(\mathrm{Sb}^{\mathrm{i}} \mathrm{Pr}_{3}\right)\right]$ & 2.195 & 104.35 & 2.147 & 105.3 \\
\hline$\left[\mathrm{InCl}_{3}\left(\mathrm{SbEt}_{3}\right)\right]$ & 2.166 & 103.85 & 2.126 & 104.1 \\
\hline$\left[\mathrm{InCl}_{3}\left(\mathrm{Sb}^{\mathrm{i}} \mathrm{Pr}_{3}\right)\right]$ & 2.189 & 105.478 & 2.165 & 107.02 \\
\hline$\left[\operatorname{lnI}_{3}\left(\mathrm{SbEt}_{3}\right)\right]$ & 2.169 & 102.867 & 2.137 & 103.7 \\
\hline$\left[\operatorname{lnl}_{3}\left(\mathrm{Sb}^{\mathrm{i}} \mathrm{Pr}_{3}\right)\right]$ & 2.196 & 104.220 & 2.168 & 106.2 \\
\hline
\end{tabular}

It has been theorized that widening of the $\mathrm{C}-\mathrm{Sb}-\mathrm{C}$ angles upon coordination corresponds to a greater $s$ character in the $\mathrm{Sb}-\mathrm{C}$ bond and increased $p$-character in the lone pair. The $\sigma$-donation from this lone pair upon coordination decreases electron density on the lone pair orbital, resulting in increased $\mathrm{C}-\mathrm{Sb}-\mathrm{C}$ angles and shorter $\mathrm{Sb}-\mathrm{C}$ bond distances. ${ }^{71}$ In order to test this, natural bond order (NBO) analysis was undertaken to provide a breakdown of the $s$ - and $p$-character of the $\mathrm{Sb}-\mathrm{C}$ bonds in both the 'free' stibines and the complexes (Table 5). This shows that there is significantly more s-contribution from the $\mathrm{Sb}$ atom in the $\mathrm{Sb}-\mathrm{C}$ bonds from the complexes, compared to that in 'free' $\mathrm{Sb}^{\mathrm{i}} \mathrm{Pr}_{3}$ and $\mathrm{SbEt}_{3}$. The uncoordinated stibines have $10.7-10.8 \%$ s-character, this doubles to $22-25 \%$ in the complexes, with only a slight increase in contribution from the $\mathrm{Sb}$ atom to the $\mathrm{Sb}-\mathrm{C}$ bond.

Table 5 The contributions of the Sb-C bond as determined by NBO analysis.

\begin{tabular}{|c|c|c|c|c|c|c|}
\hline & \multicolumn{2}{|c|}{ Contribution (\%) } & \multicolumn{2}{|c|}{$\begin{array}{l}\text { Breakdown of Sb } \\
\text { contributions (\%) }\end{array}$} & \multicolumn{2}{|c|}{$\begin{array}{l}\text { Breakdown of C } \\
\text { contributions (\%) }\end{array}$} \\
\hline & $\mathrm{Sb}$ & C & S & $p$ & s & $P$ \\
\hline $\mathrm{SbEt}_{3}$ & 31.18 & 68.82 & 10.70 & 89.30 & 20.47 & 79.53 \\
\hline $\mathrm{Sb}^{\mathrm{i}} \mathrm{Pr}_{3}$ & 31.11 & 68.89 & 10.89 & 89.11 & 19.01 & 80.99 \\
\hline$\left[\mathrm{BCl}_{3}\left(\mathrm{Sb}^{\mathrm{i}} \mathrm{Pr}_{3}\right)\right]$ & 34.70 & 65.30 & 23.26 & 76.74 & 17.86 & 82.14 \\
\hline$\left[\mathrm{BBr}_{3}\left(\mathrm{SbEt}_{3}\right)\right]$ & 34.76 & 65.24 & 22.81 & 77.19 & 19.64 & 80.36 \\
\hline$\left[\mathrm{BBr}_{3}\left(\mathrm{Sb}^{\mathrm{i}} \mathrm{Pr}_{3}\right)\right]$ & 34.79 & 65.21 & 23.07 & 76.93 & 17.86 & 82.14 \\
\hline$\left[\mathrm{Bl}_{3}\left(\mathrm{SbEt}_{3}\right)\right]$ & 34.85 & 65.15 & 22.67 & 77.33 & 19.73 & 80.27 \\
\hline$\left[\mathrm{Bl}_{3}\left(\mathrm{Sb}^{\mathrm{i}} \mathrm{Pr}_{3}\right)\right]$ & 34.85 & 65.15 & 22.89 & 77.11 & 17.92 & 82.08 \\
\hline$\left[\mathrm{All}_{3}\left(\mathrm{Sb}^{\mathrm{i}} \mathrm{Pr}_{3}\right)\right]$ & 35.87 & 64.13 & 24.07 & 75.93 & 17.50 & 82.50 \\
\hline$\left[\mathrm{GaCl}_{3}\left(\mathrm{Sb}^{\mathrm{i}} \mathrm{Pr}_{3}\right)\right]$ & 35.88 & 64.12 & 24.55 & 75.45 & 17.36 & 82.64 \\
\hline
\end{tabular}




\begin{tabular}{|c|c|c|c|c|c|c|}
\hline$\left[\mathrm{GaBr}_{3}\left(\mathrm{SbEt}_{3}\right)\right]$ & 35.60 & 64.40 & 23.81 & 76.18 & 19.18 & 80.81 \\
\hline$\left[\mathrm{Gal}_{3}\left(\mathrm{Sb}^{\mathrm{i} P{ }_{3}}\right)\right]$ & 35.65 & 64.35 & 24.00 & 76.00 & 17.46 & 82.54 \\
\hline$\left[\mathrm{InCl}_{3}\left(\mathrm{SbEt}_{3}\right)\right]$ & 36.06 & 63.95 & 24.68 & 75.33 & 19.00 & 81.00 \\
\hline$\left.\left[\operatorname{InCl}_{3}\left(\mathrm{Sb}^{\mathrm{i} P r}\right)_{3}\right)\right]$ & 36.13 & 63.87 & 25.11 & 74.89 & 17.26 & 82.74 \\
\hline$\left[\operatorname{Inl}_{3}\left(\mathrm{SbEt}_{3}\right)\right]$ & 35.61 & 64.39 & 23.85 & 76.15 & 19.18 & 80.82 \\
\hline$\left[\operatorname{lnl}_{3}\left(\mathrm{Sb}^{i} \mathrm{Pr}_{3}\right)\right]$ & 35.85 & 64.15 & 24.37 & 75.63 & 17.34 & 82.66 \\
\hline
\end{tabular}

The NBO natural charges for the calculated complexes, in addition to calculations of the individual metal halides and uncoordinated stibines (Table 6), show that the halide present on the Group 13 element (M) has a significant effect on the NBO charge density of the $\mathrm{M}$ atoms, in the order $\mathrm{Cl}>\mathrm{Br}>\mathrm{I}$, which increases down Group 13, (B < Al $<\mathrm{Ga}<\mathrm{In}$ ). Upon coordination, the NBO natural charge on the Sb-centre increases from the 'free' stibine in the order $B>A l>G a>I$, while the natural charge on $M(B, A l, G a, I n)$ atom decreases. The large positive increase of the charge on antimony upon coordination with $\mathrm{BX}_{3}$, suggests a greater degree of donation than towards other Group 13 halides, while the significant negative decrease of the charge of $\mathrm{M}$ upon coordination confirms that $\mathrm{BX}_{3}$ is a stronger electron acceptor than $\mathrm{Al}, \mathrm{Ga}$ and $\mathrm{In}$ halides.

Table 6 Showing the NBO natural charges of the complexes compared to the individual components $\mathrm{MX}_{3}$ or the $\mathrm{SbR}_{3}$.

\begin{tabular}{|c|c|c|c|c|}
\hline & \multicolumn{2}{|c|}{$\left[\mathrm{MX}_{3}\left(\mathrm{SbR}_{3}\right)\right]$} & \multirow{2}{*}{$\frac{\mathrm{SbR}_{3}}{\mathrm{Sb}}$} & \multirow{2}{*}{$\frac{\mathrm{MX}_{3}}{M}$} \\
\hline & $S b$ & $M$ & & \\
\hline$\left[\mathrm{BCl}_{3}\left(\mathrm{Sb}^{\mathrm{i}} \mathrm{Pr}_{3}\right)\right]$ & 1.746 & 0.049 & 1.141 & 0.565 \\
\hline$\left[\mathrm{BBr}_{3}\left(\mathrm{Sb}^{\mathrm{i}} \mathrm{Pr}_{3}\right)\right]$ & 1.756 & 0.291 & 1.141 & \multirow{2}{*}{0.233} \\
\hline$\left[\mathrm{BBr}_{3}\left(\mathrm{SbEt}_{3}\right)\right]$ & 1.757 & -0.291 & 1.133 & \\
\hline$\left[\mathrm{Bl}_{3}\left(\mathrm{Sb}^{\mathrm{i}} \mathrm{Pr}_{3}\right)\right]$ & 1.785 & -0.748 & 1.141 & \multirow{2}{*}{-0.215} \\
\hline$\left[\mathrm{Bl}_{3}\left(\mathrm{SbEt}_{3}\right)\right]$ & 1.777 & -0.742 & 1.133 & \\
\hline$\left[\mathrm{All}_{3}\left(\mathrm{Sb}^{\mathrm{i}} \mathrm{Pr}_{3}\right)\right]$ & 1.409 & 0.310 & 1.141 & 0.663 \\
\hline$\left[\mathrm{GaCl}_{3}\left(\mathrm{Sb}^{\mathrm{i}} \mathrm{Pr}_{3}\right)\right]$ & 1.363 & 0.989 & 1.141 & 1.286 \\
\hline$\left[\mathrm{GaBr}_{3}\left(\mathrm{SbEt}_{3}\right)\right]$ & 1.375 & 0.784 & 1.133 & 1.085 \\
\hline$\left[\mathrm{Gal}_{3}\left(\mathrm{Sb}^{\mathrm{i}} \mathrm{Pr}_{3}\right)\right]$ & 1.384 & 0.399 & 1.141 & 0.686 \\
\hline$\left[\mathrm{InCl}_{3}\left(\mathrm{Sb}^{\mathrm{i}} \mathrm{Pr}_{3}\right)\right]$ & 1.284 & 1.180 & 1.141 & \multirow[t]{2}{*}{1.404} \\
\hline$\left[\operatorname{lnCl}_{3}\left(\mathrm{SbEt}_{3}\right)\right]$ & 1.285 & 1.171 & 1.133 & \\
\hline$\left[\operatorname{lnl}_{3}\left(\mathrm{Sb}^{\mathrm{i}} \mathrm{Pr}_{3}\right)\right]$ & 1.300 & 0.719 & 1.141 & \multirow[t]{2}{*}{0.951} \\
\hline$\left[\operatorname{lnI}_{3}\left(\mathrm{SbEt}_{3}\right)\right]$ & 1.309 & 0.708 & 1.133 & \\
\hline
\end{tabular}

The calculated molecular orbitals (Figure 5) show that for $\mathrm{X}=\mathrm{Cl}$ or $\mathrm{Br}$ the HOMO lies in the $\mathrm{Sb}-\mathrm{M}$ bond in all calculated structures (SI Table S8). However, for $\left[\mathrm{Bl}_{3}\left(\mathrm{SbR}_{3}\right)\right],\left[\mathrm{Gal}_{3}\left(\mathrm{Sb}^{\mathrm{i}} \mathrm{Pr}_{3}\right)\right]$ and $\left[\operatorname{InI}_{3}\left(\mathrm{SbR}_{3}\right)\right]$ calculations show the HOMO lies on the $p$-orbitals of the iodine atoms, while the HOMO-1 is the Sb-M bonding orbital, at a slightly lower energy of 7-11 eV. The LUMO in each case is an anti-bonding orbital around the Sb-M bond.

\begin{tabular}{|l|l|l|l|}
\hline & HOMO-1 & HOMO & LUMO \\
\hline
\end{tabular}




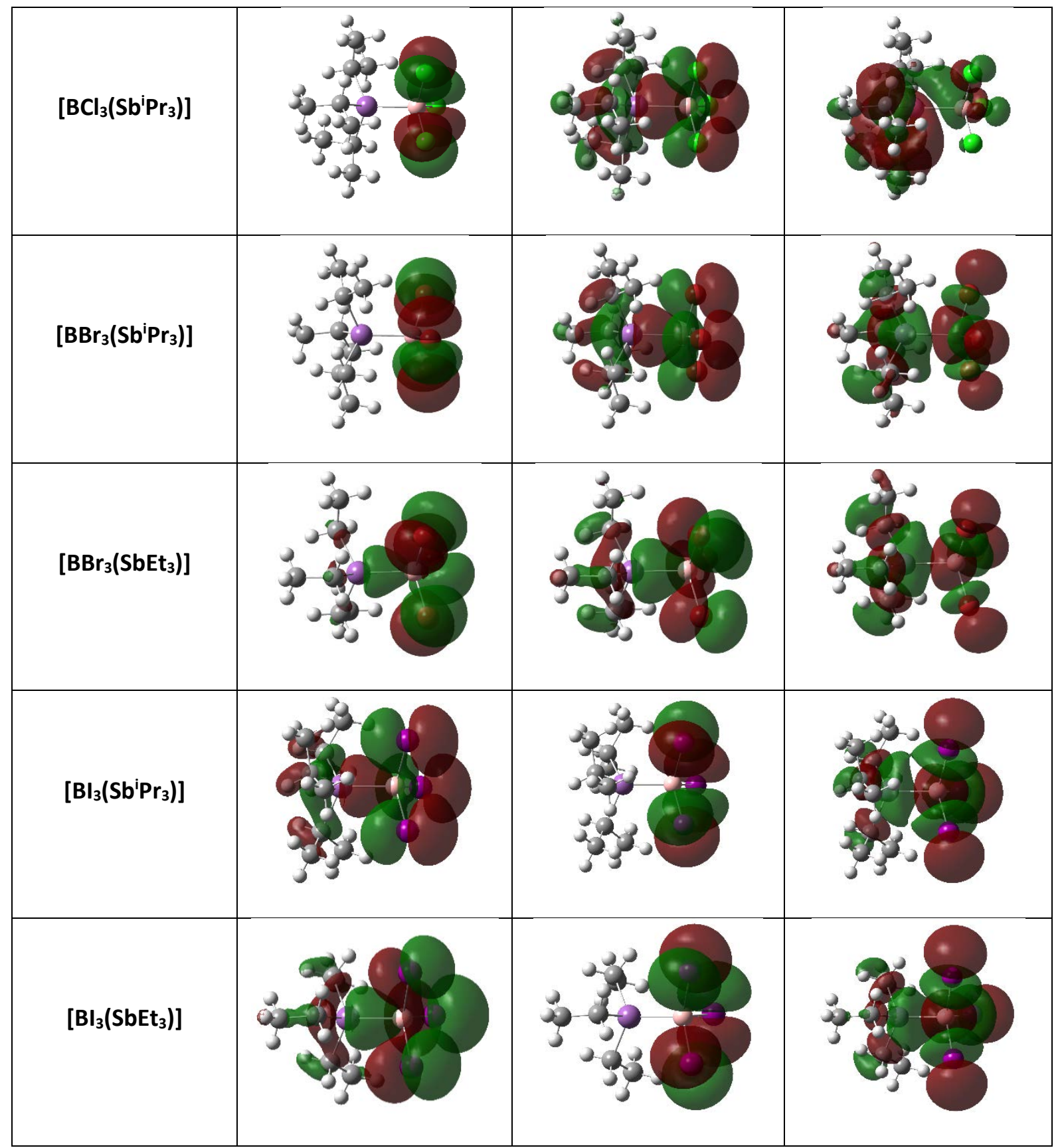

Figure 5 Frontier molecular orbitals from the $\mathrm{NBO}$ analysis of $\left[\mathrm{BX}_{3}\left(\mathrm{SbR}_{3}\right)\right]$ complexes.

In the X-ray structures eclipsed structures were found for the $\left[\operatorname{InCl}_{3}\left(\mathrm{SbEt}_{3}\right)\right],\left[\mathrm{GaBr}_{3}\left(\mathrm{SbEt}_{3}\right)\right]$ and $\left.\left[\operatorname{Inl}_{3}\left(\mathrm{SbEt}_{3}\right)\right]\right)$. However, the DFT calculations (gas phase) predict staggered confirmations for all except $\left[\operatorname{lnCl}_{3}\left(\mathrm{SbEt}_{3}\right)\right]$. The $\mathrm{X}$-ray crystal structure of $\left[\mathrm{BF}_{3}\left(\mathrm{PMe}_{3}\right)\right]$ shows an eclipsed conformation, ${ }^{25}$ whereas the calculations predict the gas phase molecule would have a staggered conformation..$^{72}$ It is probable that in all these cases, the differences between the calculated and experimental structures are a result of solid-state packing effects.

\section{Conclusions}

This work has demonstrated that trialkylstibine complexes of the Group 13 chlorides, bromides and iodides, (not Tl) can be readily synthesized with careful choice of solvents and conditions and detailed spectroscopic and structural data obtained. The data have been used to compare their properties with those of the more 
familiar phosphine and arsine analogs. Overall, the trends in Lewis acidity as revealed by the spectroscopy and structures follow those for other Group 13 pnictine complexes, with Lewis acidity trends $(\mathrm{F})<\mathrm{Cl}<\mathrm{Br}<1$ for boron, and $\mathrm{Cl}>\mathrm{Br}>\mathrm{I}$ for the three metals. Although $\mathrm{C}-\mathrm{Sb}$ bond cleavage occurs under some conditions, it is much less prevalent than would have been expected based upon the older (but sparse) literature. ${ }^{1-4}$

The significant changes in $\mathrm{C}-\mathrm{Sb}-\mathrm{C}$ angles and $\mathrm{C}-\mathrm{Sb}$ distances that occur on coordination of the stibines to the Group 13 centers have been shown by DFT calculations to be due to changes in the antimony $5 s$ and $5 p$ orbital contributions to the $\mathrm{C}-\mathrm{Sb}$ and $\mathrm{Sb}-\mathrm{M}$ bonds. Whilst similar effects have been observed on coordination of triarylstibines to d-block metal centres, ${ }^{71,73}$ the magnitude of the changes is greater in the present complexes. Further studies are needed to establish whether these differences result from the presence of aryl-C-Sb bonds (vs. alkyl-C-Sb) or to the presence of d-orbital contributions to the bonding from the transition metal.

More generally, the demonstration that a significant range of trialkylstibine complexes with Group 13 halides can be obtained may suggest that other p-block Lewis acid-stibine complexes would also be accessible. This is now the focus of our work in this area. Future work will also explore the suitability of some of the newly reported complexes as single source precursors for the deposition of GaSb and/or InSb thin film materials.

\section{Acknowledgements}

We thank the EPSRC for support through EP/K039466/1 and Professor J. M. Dyke for helpful discussions. We also thank Dr G. Stenning (ISIS, Harwell Campus, UK) for assistance with the X-ray data collection on $\left[\mathrm{Gal}_{3}\left(\mathrm{Sb}^{\mathrm{i}} \mathrm{Pr}_{3}\right)\right]$.

\section{Conflict of Interest}

The authors declare no competing financial interest.

\section{Supporting Information}

The Supporting Information is available free of charge on the ACS Publications website at DOI: XXXXXX. Crystallographic and computational details, full NMR and IR spectroscopic data for the new compounds.

\section{References}

(1) Champness, N. R.; Levason, W. Coordination chemistry of stibine and bismuthine ligands, Coord. Chem. Rev., 1994, 133, 115.

(2) Levason, W.; Reid, G. Acyclic arsine, stibine and bismuthine ligands. Comprehensive Coordination Chemistry II, McCleverty, J. A.; Meyer, T. J. Eds.; Elsevier, Oxford 2004, vol 1, p 377.

(3) Levason, W.; Reid, G. Developments in the coordination chemistry of stibine ligands. Coord. Chem. Rev., 2006, 250, 2565. 
(4) Schulz, S. The chemistry of Group $13 / 15$ compounds (III-V compounds) with the higher homologues of Group 15, Sb and Bi. Coord. Chem. Rev., 2001, 215, 1.

(5) Burt, J.; Levason, W.; Reid, G. Coordination chemistry of the main group elements with phosphine, arsine and stibine ligands. Coord Chem. Rev., 2014, 260, 65.

(6) Benjamin, S. L.; Reid, G. Neutral organoantimony(III) and organobismuth(III) ligands as acceptors in transition metal complexes - Role of substituents and co-ligands. Coord. Chem. Rev., 2015, 297-298, 168.

(7) Rat, C. J.; Silvestru, C.; Breunig, H. J. Hypervalent organoantimony and -bismuth compounds with pendant arm ligands. Coord. Chem. Rev., 2013, 257, 818.

(8) Benjamin, S. L.; Levason, W.; Reid, G.; Warr, R. P. Halostibines $\mathrm{SbMeX}_{2}$ and $\mathrm{SbMe}_{2} \mathrm{X}$ : Lewis Acids or Lewis Bases? Organometallics, 2012, 31, 1025.

(9) Benjamin, S. L.; Levason, W.; Light, M. E.; Reid, G.; Rogers, S. M. Bromostibine Complexes of Iron(II): Hypervalency and Reactivity. Organometallics, 2014, 33, 2693.

(10)Brown, M. D.; Levason, W.; Reid, G.; Webster, M. Synthesis and properties of $\operatorname{Rh}(I)$ and $\operatorname{Ir}(I)$ distibine complexes with organometallic co-ligands. Dalton Trans., 2006, 4039.

(11)Jones, J. S.; Wade, C. S.; Gabbaï, F. P. Guilty on Two Counts: Stepwise Coordination of Two Fluoride Anions to the Antimony Atom of a Noninnocent Stibine Ligand. Organometallics, 2015, 34, 2647.

(12)Sahu, S.; Gabbaï, F. P. Photoreductive Elimination of Chlorine from Antimony in an [SbPd]VII Complex. J. Am. Chem. Soc., 2017, 139, 5035.

(13)Yang, Y.; Gabbaï, F. P. Activation of a Hydroamination Gold Catalyst by Oxidation of a RedoxNoninnocent Chlorostibine Z-Ligand. J. Am. Chem. Soc., 2015, 137, 13425.

(14)Jones, J. S.; Wade, C. R.; Yanga, M.; Gabbaï, F. P. On the coordination non-innocence of antimony in nickel(II) complexes of the tetradentate $\left(\mathrm{o}-\left(\mathrm{Ph}_{2} \mathrm{P}\right) \mathrm{C}_{6} \mathrm{H}_{4}\right)_{3} \mathrm{Sb}$ ligand. Dalton Trans., 2017, 46, 5598.

(15)Werner, H. The Way into the Bridge: A New Bonding Mode of Tertiary Phosphanes, Arsanes, and Stibanes. Angew. Chem. Int. Ed., 2004, 43, 938.

(16)Benjamin, S. L.; Krämer, T.; Levason, W.; Light, M. E.; Macgregor, S. A.; Reid, G. $\left[\operatorname{Pd}_{4}\left(\mu_{3^{-}}\right.\right.$ $\left.\left.\mathrm{SbMe}_{3}\right)_{4}\left(\mathrm{SbMe}_{3}\right)_{4}\right]$ : A Pd(0) Tetrahedron with $\mu 3$-Bridging Trimethylantimony Ligands. J. Am. Chem. Soc., 2016, 138, 6964.

(17)Aldridge, S.; Downs, A. J. Eds., The group 13 metals aluminium, gallium, indium and thallium, Wiley, N.Y., 2011.

(18)Levason, W.; Reid, G.; Zhang, W. The chemistry of the p-block elements with thioether, selenoether and telluroether ligands. Dalton Trans., 2011, 40, 8491.

(19)McCleverty, J. A.; Meyer, T. J., Eds., Comprehensive Coordination Chemistry II, Elsevier, Oxford, 2004, vol. 3. 
(20)Gurnani, C.; Levason, W.; Ratnani, R.; Reid, G.; Webster, M. Synthesis, characterisation and structures of thio-, seleno- and telluro-ether complexes of gallium(III). Dalton Trans., 2008, 6274.

(21)Bessac, F.; Frenking, G. Why Is $\mathrm{BCl}_{3}$ a Stronger Lewis Acid with Respect to Strong Bases than $\mathrm{BF}_{3}$ ? Inorg. Chem., 2003, 42, 7990.

(22)Bessac, F.; Frenking, G.; Chemical Bonding in Phosphane and Amine Complexes of Main Group Elements and Transition Metals. Inorg. Chem., 2006, 45, 6956.

(23)Plumley, A. J.; Evanseck, D. Periodic trends and index of boron Lewis acidity. J. Phys. Chem. A, 2009, 113, 5985.

(24)Sivaev, I. B.; Bregadze V. I. Lewis acidity of boron compounds. Coord Chem. Rev., 2014, $270 / 271,75$.

(25) Burt, J.; Emsley, J. W.; Levason, W.; Reid, G.; Tinkler, I. S. Systematics of $\mathrm{BX}_{3}$ and $\mathrm{BX}_{2}{ }^{+}$ Complexes $(\mathrm{X}=\mathrm{F}, \mathrm{Cl}, \mathrm{Br}, \mathrm{I})$ with Neutral Diphosphine and Diarsine Ligands. Inorg. Chem., 2016, 55, 8852.

(26) Burt, J.; Levason, W. Light, M. E.; Reid, G. Phosphine complexes of aluminium(III) halides preparation and structural and spectroscopic systematics. Dalton Trans., 2014, 43, 14600.

(27) Timoshkin, A. Y.; Bodensteiner, M.; Sevastianova, T. N.; Lisovenko, A. S.; Davydova, E. I.; Scheer, M.; Grassi, C.; Butlak, A. V. Do Solid-State Structures Reflect Lewis Acidity Trends of Heavier Group 13 Trihalides? Experimental and Theoretical Case Study. Inorg. Chem., 2012, $51,11602$.

(28) Bhalla, R.; Levason, W.; Luthra, S. K.; McRobbie, G.; Monzittu, F. M.; Palmer, J.; Reid, G.; Sanderson, G.; Zhang, W. Hydrothermal synthesis of Group 13 metal trifluoride complexes with neutral N-donor ligands. Dalton Trans., 2015, 44, 9569.

(29)Bhalla, R.; Burt, J.; Hector, A. L.; Levason, W.; Luthra, S. K., McRobbie, G.; Monzittu, F. M.; Reid, G.; Complexes of aluminium, gallium and indium trifluorides with neutral oxygen donor ligands: Synthesis, properties and reactions. Polyhedron, 2016, 106, 65.

(30) Okio, C. K. Y. A.; Levason, W.; Monzittu, F. M.; Reid, G. Complexes of $\mathrm{BX}_{3}$ with $\mathrm{EMe}_{2}(\mathrm{X}=\mathrm{F}, \mathrm{Cl}$, $\mathrm{Br}, \mathrm{l} ; \mathrm{E}=\mathrm{Se}$ or Te): Synthesis, multinuclear NMR spectroscopic and structural studies. J. Organometal. Chem., 2017, 848, 232.

(31)Okio, C. K. Y. A.; Levason, W.; Monzittu, F. M.; Reid, G. Systematics of boron halide complexes with dichalcogenoether ligands - Synthesis, structures and reaction chemistry. $J$. Organometal. Chem., 2018, 854, 140.

(32) George, K.; Jura, M.; Levason, W.; Light, M. E.; Reid, G. Thio-, seleno- and telluro-ether complexes of aluminium(III) halides: synthesis, structures and properties. Dalton Trans., 2014, 43, 3637.

(33) Denniston, M. L.; Martin, D. R. J. Inorg. Nucl. Chem., 1974, 36, 2175. 
(34)Mente, D. C.; Mills, J. L. Characterization of complexes of trimethyl group 5A bases with simple boron Lewis acids. Inorg. Chem., 1975, 14, 1862.

(35)Mente, D. C.; Mills, J. L.; Mitchell, R. E. Gas-phase calorimetry of trimethyl Group Va bases with boron Lewis acids. Inorg. Chem., 1975, 14, 123.

(36) Lube, M. S.; Wells, R. L.; White, P. S. Syntheses, characterization and crystal structures of the boron-antimony adducts $\mathrm{BX}_{3} \cdot \mathrm{Sb}\left(\mathrm{SiMe}_{3}\right)_{3} .(\mathrm{X}=\mathrm{Cl}, \mathrm{Br}$ or I). Dalton Trans., 1997, 285.

(37) Takashi, Y.; Aishima, I. Organometallic complexes containing antimony and aluminum : I. Complex formation of alkylantimony compouunds with aluminum compounds. $J$. Organometal. Chem., 1967, 8, 209.

(38)Manulkin, Z. M.; Tatarenko, A. N. Cleavage of radicals from metalloörganic compounds. VIII. Cleavage of radicals by action of aluminum chloride and ferric chloride on triphenylbismuth and triphenylantimony. Zh. Obsch. Khim., 1951, 21, 93.

(39) Hering, C.; Lehmann, M.; Schulz, A.; Villinger, A. Chlorine/Methyl Exchange Reactions in Silylated Aminostibanes: A New Route To Stibinostibonium Cations. Inorg. Chem., 2012, 51, 8212.

(40) Taylor, W.; Soto, U. H.; Lynch, V. M.; Rose, M. J. Antimony-Supported $\mathrm{Cu}_{4} \mathrm{I}_{4}$ Cuboid with Short Cu-Cu Bonds: Structural Premise for Near-Infrared Thermoluminescence. Inorg. Chem., 2016, 55, 3206.

(41) Sheldrick, G. M. A short history of SHELX. Acta Crystallogr. Sect. A, 2008, 64, 112.

(42) Gaussian 09, Revision C.01, Frisch, M. J.; Trucks, G. W.; Schlegel, H. B.; Scuseria, G. E.; Robb, M. A.; Cheeseman, J. R.; Scalmani, G.; Barone, V.; Mennucci, B.; Petersson, G. A.; Nakatsuji, H.; Caricato, M.; Li, X.; Hratchian, H. P.; Izmaylov, A. F.; Bloino, J.; Zheng, G.; Sonnenberg, J. L.; Hada, M.; Ehara, M.; Toyota, K.; Fukuda, R.; Hasegawa, J.; Ishida, M.; Nakajima, T.; Honda, Y.; Kitao, O.; Nakai, H.; Vreven, T.; Montgomery, Jr., J. A.; Peralta, J. E.; Ogliaro, F.; Bearpark, M.; Heyd, J. J.; Brothers, E.; Kudin, K. N.; Staroverov, V. N.; Keith, T.; Kobayashi, R.; Normand, J.; Raghavachari, K.; Rendell, A.; Burant, J. C.; Iyengar, S. S.; Tomasi, J.; Cossi, M.; Rega, N.; Millam, J. M.; Klene, M.; Knox, J. E.; Cross, J. B.; Bakken, V.; Adamo, C.; Jaramillo, J.; Gomperts, R.; Stratmann, R. E.; Yazyev, O.; Austin, A. J.; Cammi, R.; Pomelli, C.; Ochterski, J. W.; Martin, R. L.; Morokuma, K.; Zakrzewski, V. G.; Voth, G. A.; Salvador, P.; Dannenberg, J. J.; Dapprich, S.; Daniels, A. D.; Farkas, O.; Foresman, J. B.; Ortiz, J. V.; Cioslowski, J.; Fox, D. J. Gaussian, Inc., Wallingford CT, 2010.

(43) Becke, A. D. Density-functional thermochemistry. III. The role of exact exchange. J. Chem. Phys., 1993, 98, 5648.

(44) NBO Version 3.1, Glendening, E. D.; Reed A. E.; Carpenter J. E.; Weinhold, F.

(45) Mason, J. Multinuclear NMR, Plenum, N.Y., 1987. 
(46) Aubauer, C.; Davidge, K.; Klapötke, T. M.; Mayer, P.; Piotrowski, H.; Schulz, A. Crystal Structures of the Phosphorus-Boron Adducts $\mathrm{n}-\mathrm{Pr}_{3} \mathrm{P} \cdot \mathrm{BBr}_{3}$ and $\mathrm{I}_{3} \mathrm{P} \cdot \mathrm{BBr}_{3}$. Z. Anorg. Allg. Chem., $2000,626,2373$.

(47) Nöth, H.; Wrackmeyer, B. Nuclear magnetic resonance spectroscopy of boron compounds, Springer-Verlag, N.Y., 1978.

(48) Monkowius, U.; Nogai, S.; Schmidbauer, H. Trivinylphosphineborane and related compounds. Dalton Trans., 2003, 987.

(49) Dornhaus, E.; Bolte, M.; Lerner, H. W.; Wagner, M. Phosphanylborohydrides: First Assessment of the Relative Lewis Basicities of $\left[\mathrm{BH}_{3} \mathrm{PPh}_{2}\right]^{-}, \mathrm{CH}_{3} \mathrm{PPh}_{2}$, and $\mathrm{HPPh}_{2}$. Eur. J. Inorg. Chem., 2006, 1777.

(50) Drake, J. E.; Khasrou, L. N.; Majid, A. A spectroscopic investigation of trimethyl- and triphenyl-arsine adducts of boron trihalides. Can. J. Chem., 1981, 59, 2417.

(51)Black, D. L.; Taylor, R. C. The crystal and molecular structures of the trimethylphosphineboron trihalide complexes: $\left(\mathrm{CH}_{3}\right)_{3} \mathrm{PBCl}_{3},\left(\mathrm{CH}_{3}\right)_{3} \mathrm{PBBr}_{3}$ and $\left(\mathrm{CH}_{3}\right)_{3} \mathrm{PBI}_{3}$. Acta Crystallogr. Sect. B, $1975,31,1116$.

(52) Chadha, R. K.; Chehayber, J. M.; Drake, J. E. The structures of three trimethylarsine-boron trihalide adducts: $\left(\mathrm{CH}_{3}\right)_{3} \mathrm{AsBCl}_{3},\left(\mathrm{CH}_{3}\right)_{3} \mathrm{AsBBr}_{3}$, and $\left(\mathrm{CH}_{3}\right)_{3} \mathrm{AsBl}_{3}$. J. Crystallogr. Spectros. Res., $1985,15,53$.

(53) Codero, B.; Gomez, V.; Platero-Prats, A. E.; Peves, M.; Echeverria, J.; Cremades, E.; Barragan, F.; Alvarez, S. Covalent radii revisited. Dalton Trans., 2008, 2832.

(54) Blom, R.; Haaland, A.; Seip, R. The Molecular Structures of Trimethylarsenic, Trimethylantimony and Dimethyltellurium Determined by Gas Electron Diffraction. Acta Chem. Scand. Sect. A, 1983, 37, 595.

(55)Heimann, S.; Kuczkowski, A.; Bläser, D.; Wölper, C.; Haack, R.; Jansen, G.; Schulz, S. Syntheses and Solid-State Structures of $\mathrm{Et}_{2} \mathrm{SbTeEt}$ and $\mathrm{Et}_{2} \mathrm{BiTeEt}$. Eur. J. Inorg. Chem., 2014, 4858.

(56) Conrad, E.; Pickup, J.; Burford, N.; McDonald, R.; Ferguson, M. J. Prototypical arsine-triel adducts $\left(\mathrm{R}_{3} \mathrm{AsEX}_{3}\right.$ for $\mathrm{E}=\mathrm{B}, \mathrm{Al}$, and Ga). Can J. Chem., 2010, 88, 797.

(57) Note that the $\delta\left({ }^{27} \mathrm{Al}\right)$ chemical shifts for the $\left[\mathrm{AlX}_{3}\left(\mathrm{AsR}_{3}\right)\right](\mathrm{X}=\mathrm{Br}$ or $\mathrm{I})$ quoted in ref. 36 were obtained in situ in $\mathrm{CH}_{2} \mathrm{Cl}_{2}$ and are in error due to unrecognized $\mathrm{Cl} / \mathrm{X}$ exchange.

(58) Clegg, W.; Norman, N. C.; Pickett, N. L. Structures of the indium trihalide bisphosphine complexes of $\left[\operatorname{InBr}_{3}\left(\mathrm{PMe}_{2} \mathrm{Ph}\right)_{2}\right]$ and $\left[\operatorname{InI}_{3}\left(\mathrm{PMePh}_{2}\right)_{2}\right]$. Acta Crystallogr. Sect. C, 1994, 50, 36.

(59) Brown, M.; Tuck, D. G.; Wells, E. J. Spectroscopic and crystallographic studies of phosphino adducts of indium(III) iodide. Can. J. Chem., 1996, 74, 1535.

(60) Baker, K. J.; Kloo, L. A.; Rickard, C. E. F.; Taylor, M. J. Adducts of $\mathrm{Gal}_{3}$ and Inl $\mathrm{I}_{3}$ with $\mathrm{Ph}_{3} \mathrm{P}$ and $\mathrm{Ph}_{3}$ As. J. Organometal. Chem., 1997, 545-546, 249. 
(61) Carty, A. J. Coordination chemistry of indium. Progr. Inorg. Chem., 1975, 19, 243.

(62) Emsley, J. The Elements, Oxford, 1989.

(63) Carter, J.C.; Jugie, G.; Enjalbert, R.; Galy, J. Structural studies of coordination compounds. A joint NQR and $x$-ray investigation of trialkylphosphine-gallium trichloride adducts. Inorg. Chem., 1978, 17, 1248.

(64) Cheng, F.; Hector, A. L.; Levason, W.; Reid, G.; Webster, M.; Zhang, W. Preparation, Characterization, and Structural Systematics of Diphosphane and Diarsane Complexes of Gallium(III) Halides. Inorg. Chem., 2007, 46, 7215.

(65) Godfrey, S. M.; Kelly, K. J.; Kramkowski, P.; McAuliffe, C. A.; Pritchard, R.G. Reaction of indium metal with $\mathrm{R}_{3} \mathrm{PI}_{2}\left(\mathrm{R}=\mathrm{Ph}, \mathrm{Pri}, \mathrm{Pr}^{\mathrm{n}}\right)$; structural characterisation of the novel 'subvalent' indium(ii) complex $\left.\operatorname{In}_{2}\right|_{4}\left(\operatorname{PPr}_{3}\right)_{2}$ which contains an $\operatorname{In}-\operatorname{In}$ bond, the four- and five-coordinate indium(iii) complex $\ln l_{3}\left(\mathrm{PPh}_{3}\right)_{2} \cdot \operatorname{Inl}_{3}\left(\mathrm{PPh}_{3}\right)$, and the tetrahedral indium(iii) complex $\operatorname{Inl}_{3}\left(\mathrm{PPr}_{3}{ }_{3}\right)$. Chem. Commun., 1997, 1001.

(66) Cheng, F.; Codgbrook, H. L.; Hector, A. L.; Levason, W.; Reid, G.; Webster, M.; Zhang, W. Gallium(III) halide complexes with phosphines, arsines and phosphine oxides - a comparative study. Polyhedron, 2007, 26, 4147.

(67) Gurnani, C.; Jura, M.; Levason, W.; Ratnani, R.; Reid, G.; Webster, M. Synthesis, characterisation and structures of thio-, seleno- and telluro-ether complexes of indium(III) halides. Dalton Trans., 2009, 1611.

(68) Cheng, F.; Friend, S. I.; Hector, A. L.; Levason, W.; Reid, G.; Webster, M. Preparation, Characterization, and Structural Systematics of Diphosphane and Diarsane Complexes of Indium(III) Halides. Inorg. Chem., 2008, 47, 9691.

(69) Odom, J. D.; Moore, T. F.; Goetze, R.; Nöth, H.; Wrackmeyer, B. Nuclear magnetic resonance studies of boron compounds : XVI. Carbon-13 studies of organoboranes: Phenylboranes and boron-substituted aromatic heterocycles. J. Organometal. Chem., 1979, 173, 15.

(70) Yasuike, S.; Nakata, K.; Qin, M.; Matsumura, M.; Kakusawa, N.; Kurita, J. Synthesis of arylboronates by boron-induced ipso-deantimonation of triarylstibanes with boron trihalides and its application in one-pot two-step transmetallation/cross-coupling reactions. J. Organometal. Chem., 2015, 788, 9.

(71) Bojan, V. R.; Fernández, E. J.; Laguna, A.; López-de-Luzuriaga, J. M.; Monge, M.; Olmos, M. E.; Puelles, R. C.; Silvestru, C. Study of the Coordination Abilities of Stibine Ligands to Gold(I). Inorg. Chem., 2010, 49, 5530.

(72) Loschen, C.; Voight, K.; Frunzke, J.; Diefenbach, A.; Diedonhofen, M.; Frenking, G.; Theoretical Studies of Inorganic Compounds. Quantum Chemical Investigations of the Phosphane Complexes $\mathrm{X}_{3} \mathrm{~B}-\mathrm{PY}_{3}$ and $\mathrm{X}_{3} \mathrm{Al}-\mathrm{PY}_{3}(\mathrm{X}=\mathrm{H}, \mathrm{F}, \mathrm{Cl} ; \mathrm{Y}=\mathrm{F}, \mathrm{Cl}, \mathrm{Me}, \mathrm{CN})$. Z. Anorg. Allg. Chem., 2002, 628, 1294. 
(73) Holmes, N. J.; Levason, W.; Webster, M. Ruthenium-(II) and -(III) complexes of a ditertiary stibine ligand. The effect of co-ordination on stibine ligand geometry. J. Chem. Soc., Dalton Trans., 1998, 3457. 
Contents Graphic:

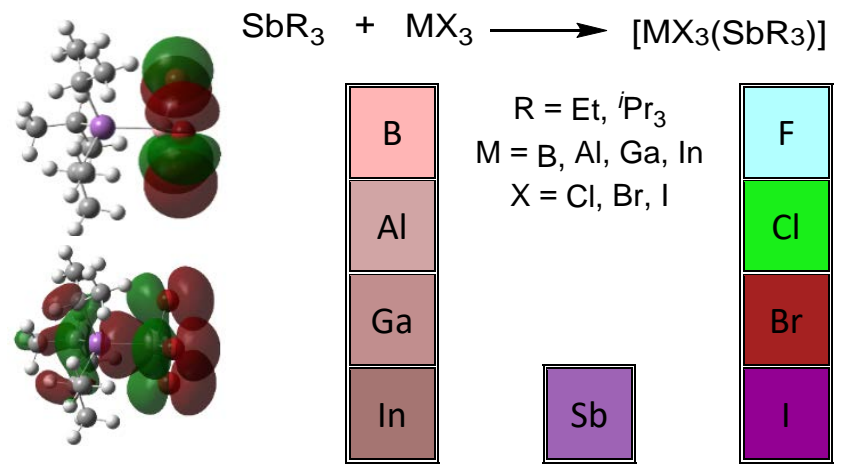

